Omega-P, Inc.

291 Whitney Avenue, Suite 401

New Haven, CT 06511

FINAL REPORT SBIR GRANT DE-FG02-07 ER 84862

\title{
HIGH-INTENSITY PROTON ACCELERATOR
}

\section{CONTENTS}

I. INTRODUCTION 2

II. TECHNICAL APPROACH - MULTI-CAVITY PROTON ACCELERATOR 3

III. TECHNICAL APPROACH - CONCEPTUAL DESIGN FOR A COUNTERPART MULTI-CAVITY ELECTRON CYCLOTRON 13

IV. ENGINEERING DESIGN AND PRELIMINARY TESTS FOR COUNTERPART MULTI-CAVITY ELECTRON CYCLOTRON ACCELERATOR 16

V. ANALYSIS AND TEST OF CAVITY WITH RADIAL VANES 26

VI. TRANSPORT OF PROTON BEAM FROM MULTI-CAVITY CYCLOTRON 30

VII. SUMMARY 33

References 37

\section{SUMMARY}

Analysis is presented for an eight-cavity proton cyclotron accelerator that could have advantages as compared with other accelerators because of its potentially high acceleration gradient. The high gradient is possible since protons orbit in a sequence of $\mathrm{TE}_{111}$ rotating mode cavities of equally diminishing frequencies with path lengths during acceleration that greatly exceed the cavity lengths. As the cavities operate at sequential harmonics of a basic repetition frequency, phase synchronism can be maintained over a relatively wide injection phase window without undue beam emittance growth. It is shown that use of radial vanes can allow cavity designs with significantly smaller radii, as compared with simple cylindrical cavities. Preliminary beam transport studies show that acceptable extraction and focusing of a proton beam after cyclic motion in this accelerator should be possible. Progress is also reported on design and tests of a four-cavity electron counterpart accelerator for experiments to study effects on beam quality arising from variations injection phase window width. This device is powered by four 500-MW pulsed amplifiers at 1500,1800,2100, and $2400 \mathrm{MHz}$ that provide phase synchronous outputs, since they are driven from a with harmonics derived from a phase-locked $300 \mathrm{MHz}$ source.

Work reported here was performed by former Omega-P senior research scientist Dr. S.Yu. Kazakov (now at FNAL), with important input from Omega-P consultant Dr. V.P. Yakovlev of FNAL and research scientists Dr. M.A. LaPointe and Dr. S.V. Shchelkunov of Yale University. Dr. J.L. Hirshfield served as Principal Investigator, and wrote this report. 
Omega-P, Inc.

Final Report, DoE SBIR Grant DE-FG02-07 ER 84862

HIGH-INTENSITY PROTON ACCELERATOR

\section{INTRODUCTION}

Under Topic 27c in the DoE 2007 SBIR Program Solicitation, proposals were sought to “...develop new methods of intense beam acceleration ... with applications as high-intensity proton drivers for neutron production, waste transmutation, energy production in nuclear reactors, medical proton therapy $(250 \mathrm{MeV})$, and radioisotope production." The Solicitation also made reference to "...design of an electron model/prototype to directly simulate operation under space-charge limiting conditions." Omega-P, Inc. submitted a proposal in response, entitled "High-Intensity Proton Accelerator" and was awarded Phase I and II grants DE-FG02-07-ER 84862. The principal goal in Phase I was to carry out further detailed analysis of a novel concept for a high-gradient accelerator of an intense proton beam in a normal conducting (i.e., room temperature) structure. The structure consists of a cascade of RF cavities in a nearly-uniform axial magnetic field. The proton energy gain mechanism relies upon cyclotron resonance acceleration in each cavity. An additional goal of the Phase I program was to devise a conceptual design for an electron counterpart of the proton accelerator, to be built and tested during Phase II. The electron counterpart machine is intended to confirm design principles used for the proton machine, and to assess issues associated with space-charge forces at high current.

Advantages of the proposed multi-cavity high-intensity proton accelerator include the following, where the parameters cited correspond to the point design described below.

- high effective acceleration gradient (> $40 \mathrm{MV} / \mathrm{m})$;

- high average current (>100 mA);

- low surface electric fields in the cavities (<6 MV/m);

- low rms energy spread for the accelerated beam $(<0.1 \%)$;

- large apertures in the cavities, to guard against beam interception and HOM wake fields;

- absence of any need for additional focusing magnets; and

- use of room-temperature RF cavities.

Against these positive attributes for the multi-cavity proton cyclotron, it is necessary to balance its need for a large superconducting solenoid: the 8-T large diameter magnet taken in the point design described below is within the present state of the art [7], but would undoubtedly be costly. A second item to balance against the listed positive attributes is the less-than-ideal RFto-beam power efficiency $\eta$ due to the use of room-temperature cavities, where $\eta=69 \%$ in the cited point design. These factors must eventually be weighed in comparing the multi-cavity proton cyclotron with an alternative, such as a superconducting proton linac having lower acceleration gradient and thus greater length. However, before an informed examination of competitive issues can be initiated, a greater understanding of the acceleration process should be at hand, through studies such as that described in this report.

Availability of intense GeV-range proton beams would allow extension of basic research and applications into beam intensity ranges that have been heretofore inaccessible. For example, the new proton accelerator concept presented here could form the basis for an alternative to a superconducting linac for production of high-intensity proton beams, either for a K-factory or a $\Pi$-factory, in a muon collider, or in a neutron spallation source such as that needed for driving a sub-critical reactor for accelerator-based transmutation of nuclear waste (ATW). Development of a viable ATW system could, by transmutation of actinides, allow reduction of the half-life in 


\section{Omega-P, Inc.}

Final Report, DoE SBIR Grant DE-FG02-07 ER 84862

HIGH-INTENSITY PROTON ACCELERATOR

present nuclear waste to be shortened to about 500 years, rather than 500,000 years, and thus render much more practical the oversight of waste storage - either on-site or at Yucca Mountain type repository. The two most powerful proton accelerators in use at present are the $800 \mathrm{MeV}$, $1.5 \mathrm{~mA}$ linac at Los Alamos National Laboratory, and the $590 \mathrm{MeV}, 1.8 \mathrm{~mA}$ cyclotron at Paul Scherer Institute. Both have much lower beam power than the accelerator discussed in this proposal. Distinctive attributes of the new accelerator concept include use of normal-conducting cavities (but a superconducting magnet); a high intrinsic acceleration gradient, with a corresponding short length; modest surface fields in the cavities; large beam apertures in the cavities; no need for focusing magnets between cavities; no need for an accumulator ring to build up bunch charge; and intrinsic scanning of the beam over a target for tailored distribution of dose and waste heat. Admittedly, cost issues attach to the need for a large superconducting solenoid; care would be required to control magnet quenches given the GJ-level stored magnetic energy; and power in cavity ohmic losses $\left(<100 \mathrm{~W} / \mathrm{cm}^{2}\right)$ would need to be sensibly dissipated. Since cavity frequencies scale in direct proportion to magnetic field strength, the availability of a field stronger than the $8 \mathrm{~T}$ in the example shown would reduce the radius and length of the machine in approximate inverse proportion to the field strength. Preliminary studies indicate that some reduction in cavity radii can also be achieved by using radial vanes inserted into the cavities. It was shown that a vaned cavity can have a shunt impedance similar to that of a simple cylinder. Clearly, analysis so far suggests that the new proton accelerator has technical promise, with a number of distinctive benefits to the scientific and general public. But continued analysis, together with laboratory evaluation of an electron counterpart, are required before a fullyinformed judgement can be made as to its competitive merits. These latter steps form the basis of the program proposed here.

An April 2007 presentation [10] by R. Raja of FNAL reviews the thorium cycle as the basis for an accelerator-driven sub-critical reactor working as an energy amplifier, as suggested over a decade ago by Carlo Rubbia. In Raja's presentation, need for development of an effective $10-\mathrm{MW}, 1 \mathrm{GeV}$ proton driver is stressed. It is for that application, among others listed above, that the work towards evolving a multi-cavity proton cyclotron is herein proposed. Benefits to society from the realization of a sub-critical reactor power plant with the large margin of safety that is possible with a proton driver could be substantial indeed.

\section{TECHNICAL APPROACH - MULTI-CAVITY PROTON ACCELERATOR}

The new proton accelerator concept that Omega-P scientists have analyzed and patented [2-6] is based on use of a cascade of rotating-mode normal conducting $\mathrm{TE}_{111}$ cavities in a strong nearly-uniform static magnetic field. Cyclotron-resonance acceleration in each cavity provides energy gain for the protons. The cavity resonance frequencies $f_{n}$ decrease from $f_{1}$ for the first cavity, with a fixed frequency interval $\Delta f$ between each of the $N$ cavities; thus $f_{n}=f_{1}-(n-1) \Delta f$ and $f_{1}=\ell \Delta f$, with the integer $\ell \geq n$, and $1 \leq n \leq N$. Proton pulses injected into the first cavity at intervals $\Delta T=1 / \Delta f$ or integer multiples thereof can experience continuous energy gain throughout the entire cavity cascade, provided the relative phases for fields in each cavity are properly adjusted. Since protons can execute a number of orbital turns in each cavity well in excess of unity, voltage gain can be much larger than the product of peak electric field and cavity length. In light of this latter feature, cavity surface fields can be well below 
breakdown and multipactor limits, even for substantial proton energy gain per cavity. Furthermore, since protons are strongly restrained to follow spiral orbits in the strong static magnetic field, additional focusing magnets are not needed, thereby simplifying the design, conserving space, and not diluting the effective acceleration gradient. In addition to these distinctive features, it was discovered that this acceleration mechanism can be sustained for exceptionally wide injection phase angles in the first cavity, e.g., two rf cycles in the example shown below. This feature is highly significant, in that it allows operation with high duty factor $(\sim 13 \%)$ and low peak proton current $(<1 \mathrm{~A})$, thereby mitigating against issues that can arise from high beam space charge and yet seem to allow high average proton current $(>10 \mathrm{~mA})$.

A schematic diagram to illustrate the accelerator concept is shown in Fig. 1. The diagram shows that, after the accelerated protons pass through the cascade of cavities ( 8 in this example), they emerge on trajectories that fan out in the diverging magnetic field; these trajectories scan azimuthally with time at the gyrofrequency. As a consequence, the power deposition profile on the target can be tailored by judicious design of the magnetic field profile. This allows the very substantial power of the beam to be "self-scanned" over a large internal area on a cylindrical spallation target within, for example, a sub-critical reactor or an assembly of spent fuel rods, thereby producing uniform neutron flux and allowing manageable heat dissipation. Alternately, depending upon the magnetic field geometry, the protons can be transported to a remote target. Practical means for achieving this invariably necessary step are discussed in Section III.

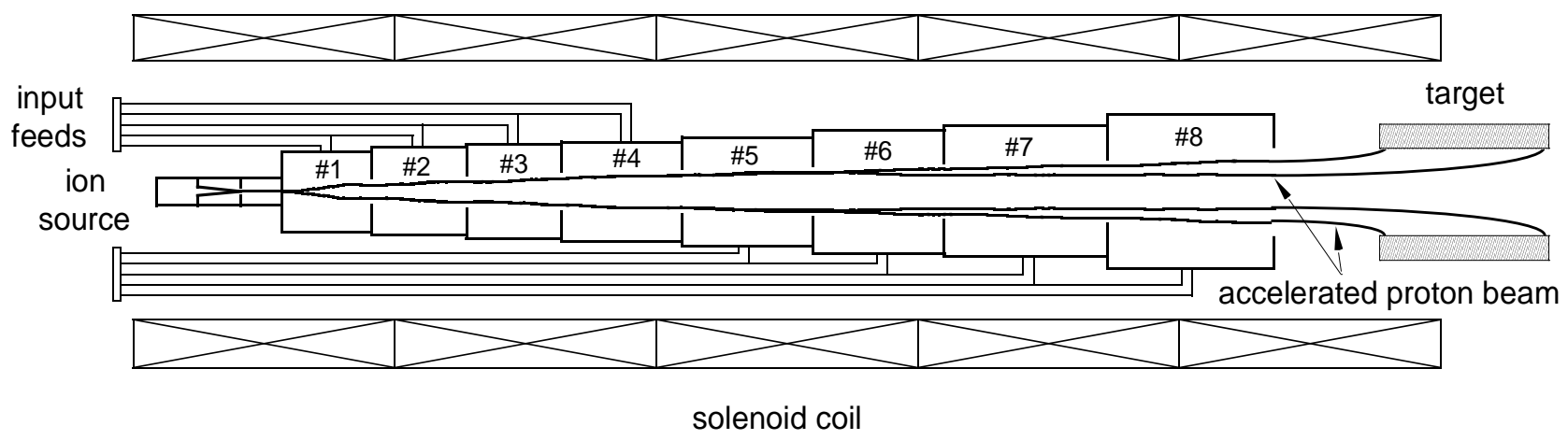

Fig. 1. Schematic of the multi-cavity proton cyclotron, with eight cavities in this example.

Basic analysis to model the multi-cavity proton cyclotron invokes the following assumptions:

a. The beam's duty factor was taken to be $1 / 7.5$, corresponding to an injection phase at the first cavity of $720^{\circ}$. For an average current of $122 \mathrm{~mA}$, this corresponds to a peak injected current of $915 \mathrm{~mA}$ (at $1000 \mathrm{kV}$ ). For this peak current in the strong guide magnetic field of the machine, the influence of space charge forces on proton dynamics can be shown to be small.

$b$. An eight-cavity structure with 20 -cm long beyond-cut-off drift tunnels between cavities was analyzed for a zero-emittance injected proton beam. In this model, the individual cavity fields were assumed to have idealized forms that neglect apertures. 


\section{Omega-P, Inc.}

Final Report, DoE SBIR Grant DE-FG02-07 ER 84862

\section{HIGH-INTENSITY PROTON ACCELERATOR}

c. Then removing the assumption of a zero-emittance beam, initial parameters were taken to correspond to a $915 \mathrm{~mA}, 1000 \mathrm{keV}$ Brillioun beam in an 8-T magnetic field, i.e. to a "matched" beam, as a reasonable means to test the sensitivity of the acceleration mechanism to finite spreads in energy and velocity, and to finite beam radius. Almost no degradation in accelerator performance was found for this beam model.

$d$. To test the assumption of using idealized cavity fields in the analysis, an Omega-P 3-D rf simulation code for axisymmetric structures was applied to the eight-cavity cascade. From this analysis, it was found that drift tunnel lengths between several of the lower frequency cavities had to be increased to greater than $20 \mathrm{~cm}$, in order to reduce to negligible levels the leakage of fields between a cavity and its lower frequency neighbor. This increase in drift tunnel length did not lead to a significant degradation in accelerator performance, except for a small decrease in effective acceleration gradient due to the increased system length.

$e$. To test the validity of dynamical orbit solutions found using idealized cavity fields with drift tunnels between cavities, a bench-mark dynamical solution was obtained for the first three cavities using realistic fields in the cavities, including the apertures and drift tunnels, using a modified Omega-P magnicon code. Results of this test were nearly identical to results from the analysis using idealized fields.

f. An rf field simulation was carried out for a $64 \mathrm{MHz}$ cavity (the last in the eight-cavity chain) containing four profiled radial vanes. This simulation showed that a significant reduction in outer cavity radius could be achieved $(172 \mathrm{~cm} \rightarrow 120 \mathrm{~cm})$, with only a moderate reduction in transverse shunt impedance $\left(4.1 \mathrm{M} \Omega / \mathrm{m}^{2} \rightarrow 3.5 \mathrm{M} \Omega / \mathrm{m}^{2}\right)$. Since reduced cavity radius translates into reduced magnet bore, this design change should reduce considerably the cost for the superconducting magnet.

g. To test stability of the acceleration mechanism with respect to fluctuations in rf power levels, accelerator performance calculations were carried out for overall power levels $P$ that varied within $\pm 8 \%$ of the level $P_{\text {opt }}$ that optimized performance. For this exercise, the magnetic field profile, cavity and drift tunnel dimensions, injected beam parameters, and relative rf phase values were all held constant. This study was to allay concerns that deleterious beam reflections or instability might result from small perturbations in applied rf power. Within the power fluctuation range $0.98<P / P_{o p t}<1.08$, no beam reflection or instability resulted; the only consequence of power variation was a slight diminution in the final acceleration energy of the protons. For $P / P_{o p t}<0.98$, some reflection of protons was encountered.

As stated, analysis of the multi-cavity proton cyclotron included finite injected beam emittance and drift tunnels between cavities. Beam parameters correspond to those for an ideal Brillioun beam of radius $r_{b}[\mathrm{~m}]$ with the specified peak current $I[\mathrm{~A}]$ in the specified magnetic field $B_{z}[\mathrm{~T}]$. These parameters are related [5] by $r_{b} B_{z}=\sqrt{240 I U_{p} / \beta \gamma c^{2}}$, where $U_{p}$ is the proton rest mass expressed in $\mathrm{eV}\left(938 \times 10^{6} \mathrm{eV}\right), \gamma$ and $\beta$ are relativistic energy and velocity factors for the beam, and $c$ is speed of light in $\mathrm{m} / \mathrm{s}$. Beam parameters for the example considered are listed in Table I.

Computed accelerator performance for a cascade of eight cavities with resonance frequencies between 120 and $64 \mathrm{MHz}$, and with an 8-T axial magnetic field, is shown in Figs. 


\section{Omega-P, Inc.}

Final Report, DoE SBIR Grant DE-FG02-07 ER 84862

HIGH-INTENSITY PROTON ACCELERATOR

2-6 with parameters listed in Table II. Figs. 2 shows the gain in average proton relativistic energy factor $\gamma$ and the magnetic field profile found, that optimized the energy gain while avoiding particle reflections, and minimized energy spread for the accelerated beam. Fig. 3 shows the evolution with axial position of the normalized average transverse and axial velocities; Fig. 4 shows to scale the orbit of a typical proton; Fig. 5 shows the evolution with axial position of rms spreads in energy and axial momentum; and Fig. 6 shows the areal power dissipation profile on the cavity walls. Finally, Table II summarizes the parameters found for this example with large duty factor (1/7.5). It is found that a $1 \mathrm{MeV}, 122 \mathrm{~mA}$ beam is accelerated to $954 \mathrm{MeV}$ (average beam power $=116 \mathrm{MW}$ ), over a length of $25.2 \mathrm{~m}$, using $174 \mathrm{MW}$ of total RF power. For this example, RF-to-beam power efficiency is $67 \%$.

The field profile used here for computational convenience is composed of straight-line segments, which are of course unphysical; it is not expected that natural curvature near where the segments join will influence the results significantly. Radial field components in each segment are of course included in the analysis.

Table I. Proton beam parameters - ideal Brillouin beam.

\begin{tabular}{|c|c|}
\hline initial proton beam energy & $1000 \mathrm{keV}$ \\
\hline normalized initial proton velocity $\beta c$ & $0.04614 c$ \\
\hline peak beam current $I$ & $0.915 \mathrm{~A}$ \\
\hline average beam current $\langle I\rangle$ & $0.122 \mathrm{~A}$ \\
\hline pulse period & $125 \mathrm{nsec}$ \\
\hline pulse duration & $16.7 \mathrm{nsec}$ \\
\hline duty factor & $1 / 7.5=13.3 \%$ \\
\hline axial magnetic field $B_{z}$ & $8.0 \mathrm{~T}$ \\
\hline Brillouin radius $r_{b}$ & $0.9 \mathrm{~mm}$ \\
\hline energy depression $(30 I / \beta)\left[1+2 \ln \left(R_{w} / r_{b}\right)\right]$ & $18 \mathrm{keV}$ \\
\hline energy spread across beam $30 I / \beta$ & $600 \mathrm{eV}$ \\
\hline rms perpendicular velocity spread $c \Delta \beta_{\perp}$ & $6.5 \times 10^{-4} c$ \\
\hline relative perpendicular velocity spread $\Delta \beta_{\perp} / \beta$ & 0.0141 \\
\hline
\end{tabular}




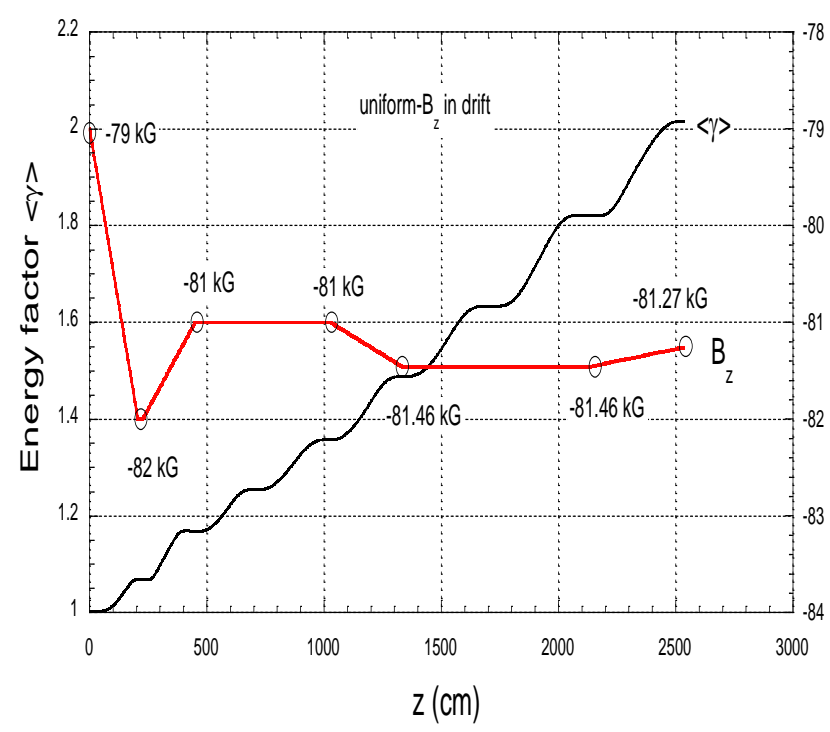

Fig. 2. Magnetic field profile, and evolution of average proton energy gain, for the example discussed in the text with duty factor of $1 / 7.5$. Field variation is about $\pm 2 \%$ around a mean value of about $8.1 \mathrm{~T}$.

Average proton energy reaches $\sim 1 \mathrm{GeV}$ in a 25 -m long machine.

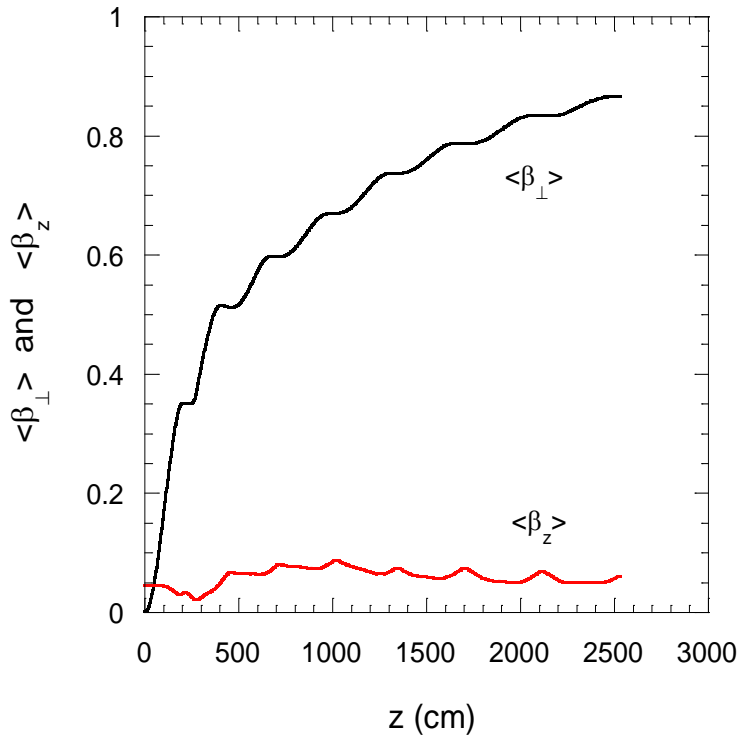

Fig. 3. Dependence of average normalized transverse velocity $\left\langle\beta_{\perp}\right\rangle$ and average normalized axial velocity $\left\langle\beta_{z}\right\rangle$ on $z$, for the example discussed in the text with duty factor of $1 / 7.5$. Stable monotonic growth in $\left\langle\beta_{\perp}\right\rangle$ is seen, and stable axial velocity is sustained, by the ponderomotive RF forces.

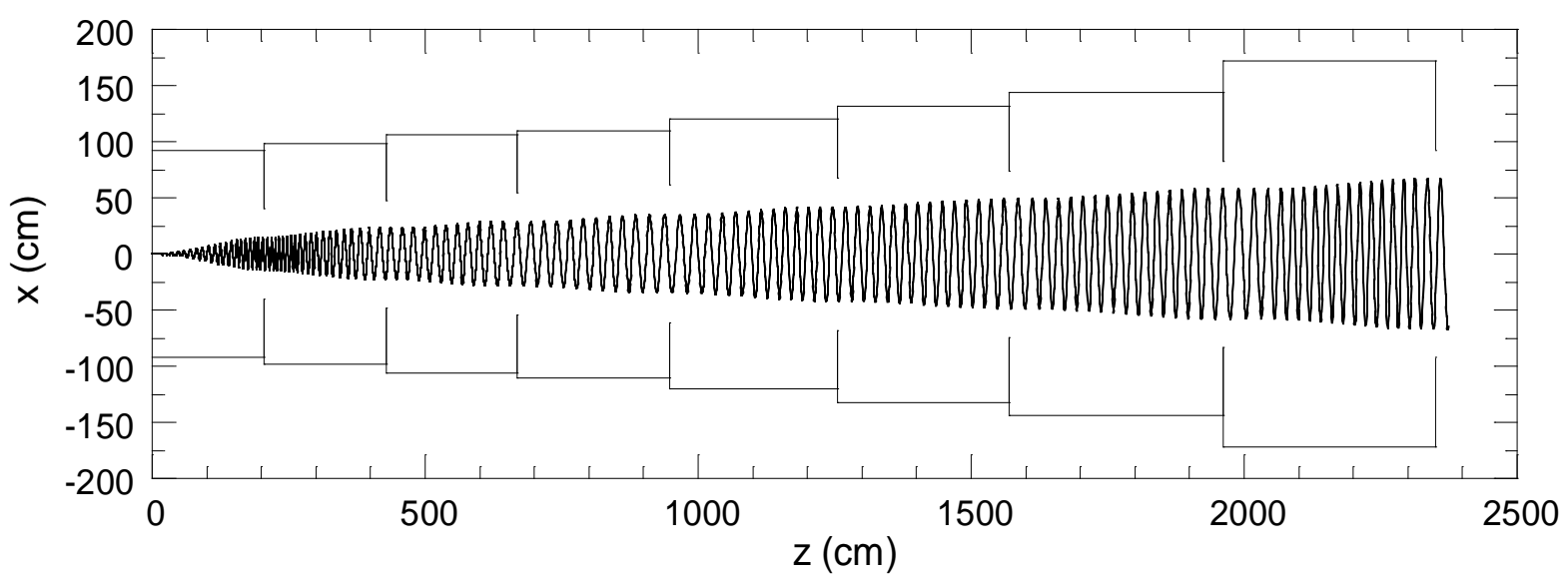

Fig. 4. Projection onto the $x$ - $z$ plane of the orbit of a typical proton, to scale. Outlines of the cavities are also shown. Since the orbit radii are considerably smaller than the cavity radii, the opportunity exists to insert radial vanes that will result in smaller outer radii for the cavities while preserving adequate apertures for the beam; this feature is discussed below. Note the substantial number of turns in each cavity. Drift tunnels between cavities are not included in this computation. 


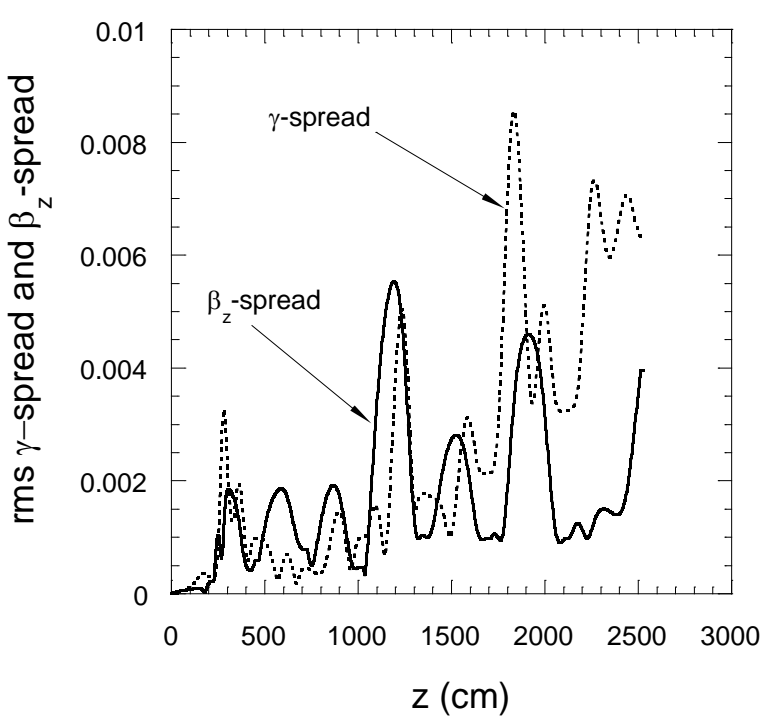

Fig. 5. Dependence of spreads in rms relativistic energy factor $\gamma$ and axial velocity $\beta_{z}$ on axial distance. Oscillatory variations are evidence for the stabilizing effects of the forces arising from the combination of RF and static magnetic fields. Energy and velocity spreads are $6 \mathrm{MeV}$ and $0.0040 c$ at the end of the device.

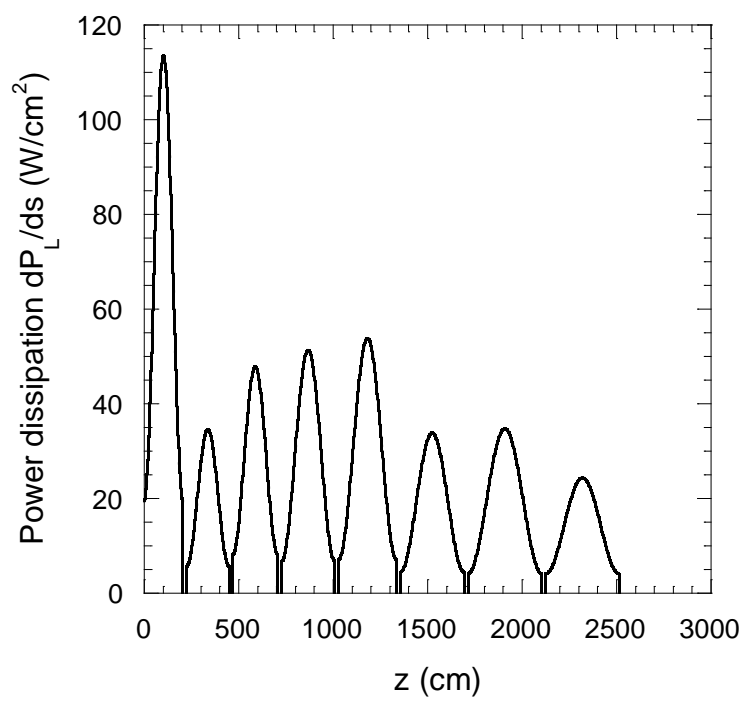

Fig. 6. Areal power dissipation in $\mathrm{W} / \mathrm{cm}^{2}$ on cylindrical cavity walls versus axial distance. Dissipation on the cavity end walls is smaller than on cylindrical walls for all cavities.

Table II. Parameters for a $122 \mathrm{~mA}$, 8-cavity proton cyclotron accelerator. Duty = 1/7.5.

\begin{tabular}{|c||c|c|c|c|c|c|c|c|c|}
\hline stage \# & $\begin{array}{c}\text { cavity } \\
\text { freq. } \\
(\mathrm{MHz})\end{array}$ & $\begin{array}{c}\text { cavity } \\
\text { radius } \\
(\mathrm{cm})\end{array}$ & $\begin{array}{c}\text { cavity } \\
\text { length } \\
(\mathrm{m})\end{array}$ & $\begin{array}{c}\mathrm{RF} \\
\text { power } \\
\text { input } \\
(\mathrm{MW})\end{array}$ & $\begin{array}{c}\text { relative } \\
\mathrm{RF} \\
\text { phase }\end{array}$ & $\begin{array}{c}\text { beam- } \\
\text { loaded } \\
\text { cavity } \\
Q_{\mathrm{L}}\end{array}$ & $\begin{array}{c}\text { intrinsic } \\
\text { cavity } Q_{\mathrm{o}} \\
\text { (copper) }\end{array}$ & $\begin{array}{c}\text { peak } \\
\text { surface } \\
\text { field } \\
(\mathrm{MV} / \mathrm{m})\end{array}$ & $\begin{array}{c}\text { mean } \\
\text { energy } \\
\text { gain } \\
(\mathrm{MeV})\end{array}$ \\
\hline \hline 1 & 120 & 92 & 2.06 & 18.0 & 0 & $6.25 \times 10^{4}$ & $1.1 \times 10^{5}$ & 7.2 & 63.6 \\
\hline 2 & 112 & 98 & 2.23 & 15.0 & $1.40 \pi$ & $2.68 \times 10^{4}$ & $1.1 \times 10^{5}$ & 4.0 & 92.9 \\
\hline 3 & 104 & 106 & 2.39 & 15.5 & $1.45 \pi$ & $4.36 \times 10^{4}$ & $1.2 \times 10^{5}$ & 4.8 & 80.9 \\
\hline 4 & 96 & 110 & 2.81 & 18.5 & $1.85 \pi$ & $4.39 \times 10^{4}$ & $1.2 \times 10^{5}$ & 4.9 & 96.1 \\
\hline 5 & 88 & 120 & 3.07 & 24.0 & $0.50 \pi$ & $4.41 \times 10^{4}$ & $1.2 \times 10^{5}$ & 5.1 & 124.3 \\
\hline 6 & 80 & 132 & 3.38 & 23.0 & $1.70 \pi$ & $3.67 \times 10^{4}$ & $1.3 \times 10^{5}$ & 4.1 & 135.3 \\
\hline 7 & 72 & 144 & 3.92 & 30.0 & $0.15 \pi$ & $3.65 \times 10^{4}$ & $1.3 \times 10^{5}$ & 4.2 & 177.0 \\
\hline 8 & 64 & 172 & 3.89 & 30.0 & $0.55 \pi$ & $3.85 \times 10^{4}$ & $1.5 \times 10^{5}$ & 3.9 & 182.7 \\
\hline \hline total & & & 25.15 & 174.0 & & & & & 952.7 \\
\hline
\end{tabular}

$*$ Total length includes 20 -cm drift tunnels. Beam power $=116 \mathrm{MW}$. Efficiency $=66.7 \%$. 


\section{Omega-P, Inc.}

Final Report, DoE SBIR Grant DE-FG02-07 ER 84862

HIGH-INTENSITY PROTON ACCELERATOR

The average acceleration gradient for the example shown in Figs. 2-6 and Table II is $37.9 \mathrm{MeV} / \mathrm{m}$, This gradient is not necessarily an upper limit; indeed, higher values would result if the RF input power levels were raised. Raised power levels could be contemplated since peak surface fields are well below multipactor and breakdown limits. Fig. 6 shows that the heat dissipation levels on the cavity walls to be accommodated are below $50 \mathrm{~W} / \mathrm{cm}^{2}$, except within a narrow band on the first cavity where the level rises to about $115 \mathrm{~W} / \mathrm{cm}^{2}$. These levels are within acceptable limits for heat dissipation on water-cooled copper surfaces.

Further analysis was carried out with the aim of testing the assumptions embodied in the above calculations which assumed that the fields of adjacent cavities could be isolated from one-another, and that the influence upon the rf fields of large cavity apertures and finitelength drift tunnels was relatively inconsequential. Sophisticated Omega-P codes used for studying electron dynamics in realistic RF structures [8], such as magnicons and accelerator structures, were modified for modeling the multi-cavity proton cyclotron. RF field analysis, without the presence of the proton beam, as well as proton dynamics calculations in the realistic fields of cavities, were carried out. Fig. 7 shows cavity contours and field plots in the cascade of eight cavities excited at the individual resonance frequencies of each cavity, to show the degree of isolation of the cavities from one another. The $20-\mathrm{cm}$ drift tubes have diameters large enough to pass the proton orbits. The field lines in Fig. 7 correspond to projections of surfaces of constant $r H_{\theta}$, a quantity used to gauge field distributions. A quantitative measure of field penetration is shown in Fig. 8, which shows the transverse $\mathrm{rf}$ magnetic field in the $7^{\text {th }}$ cavity, as a function of $z$, for two values of the drift tunnel length, 20 $\mathrm{cm}$ and $60 \mathrm{~cm}$. The $7^{\text {th }}$ cavity is seen from Fig. 7 to be the worst offender. While the field penetration is finite but small for the $20-\mathrm{cm}$ drift tunnel case, it is seen in Fig. 8 to be essentially absent for the $60-\mathrm{cm}$ case. Thus field penetration can be virtually eliminated by use of suitably long drift tunnels, with an obvious penalty in effective acceleration gradient.

Proton dynamics will also be influenced by the large apertures in the end walls, and by finite-length drift tunnels. To gauge this influence, a simulation study was undertaken in the realistic fields of the first three adjacent cavities, for comparison with the analysis that used field forms for idealized cavities. For this simulation, Brillouin beam parameters as in Table I were assumed, and compared with the case of a cold beam. Results are shown in Figs. 9 and 10 , using initial parameters for a cold beam, and the Brillouin beam, adjusted for equal values of total dissipated rf power. Energy gain values were virtually identical for the two cases. 


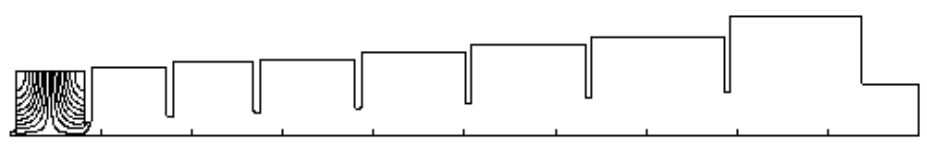

$120 \mathrm{MHz}$

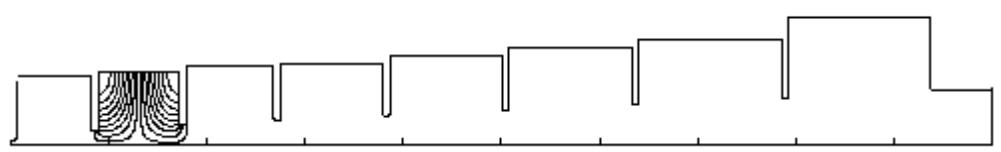

$112 \mathrm{MHz}$

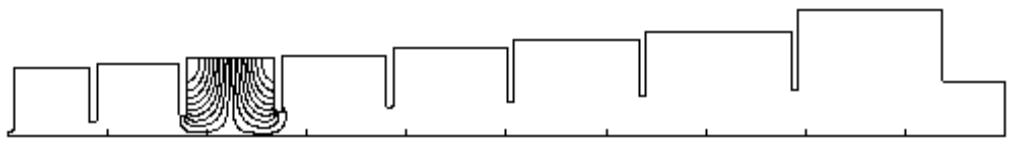

$104 \mathrm{MHz}$

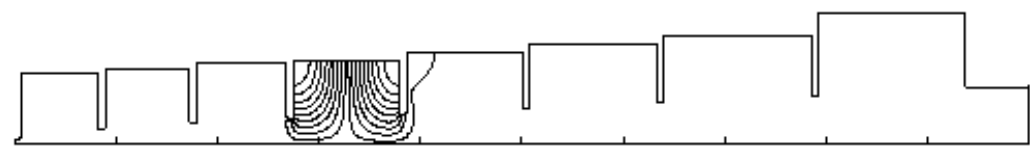

$96 \mathrm{MHz}$

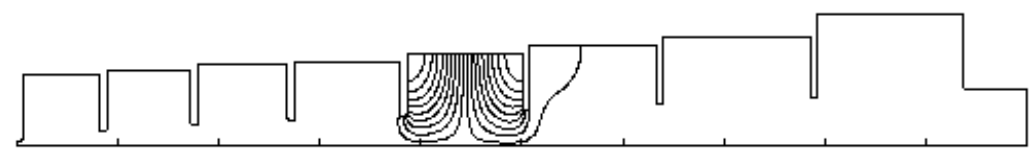

$88 \mathrm{MHz}$

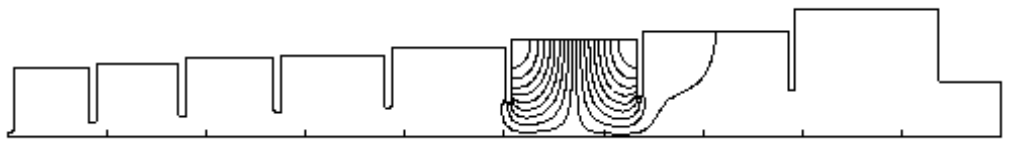

$80 \mathrm{MHz}$

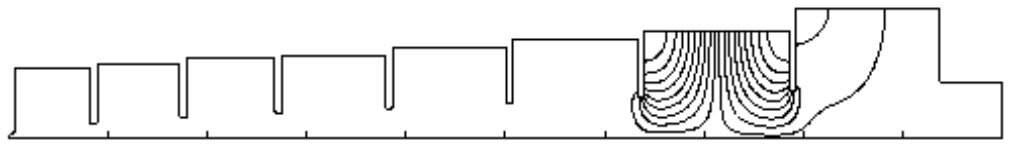

$72 \mathrm{MHz}$

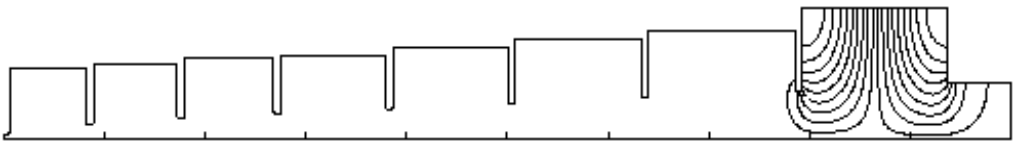

$64 \mathrm{MHz}$

Fig. 7. Field maps for the eight-cavity chain at the indicated resonant frequencies for each cavity, with 20-cm long drift tunnels between the cavities. Note the negligible "spill-over" of fields between adjacent cavities. 


\section{Omega-P, Inc.}

Final Report, DoE SBIR Grant DE-FG02-07 ER 84862

HIGH-INTENSITY PROTON ACCELERATOR

To illustrate the possibility of reactively-loading the cavities to reduce their radii while preserving the dipole nature of the $\mathrm{TE}_{111}$ modes, an example was devised for the $64-\mathrm{MHz} 8^{\text {th }}$ cavity. An RF simulation was carried out for a modified structure, one quadrant of which is shown in Fig. 11, which contains radial vanes supporting four opposing plates with ends rounded to reduce the peak surface electric field. The dipole-mode $E$-field pattern is also shown. (Rotating fields are obtained by exciting two such modes in time quadrature.) Table III gives parameters for this cavity, and for a cylindrical cavity. An aperture radius of $74 \mathrm{~cm}$ is chosen to clear the $65 \mathrm{~cm}$ orbit for the protons. Table III shows that the cavity diameter is reduced by $30 \%$, from 344 to $240 \mathrm{~cm}$, while the transverse shunt impedance* $Z_{\perp}$ is reduced by only $15 \%$, from 4.1 to $3.5 \mathrm{M} \Omega / \mathrm{m}^{2}$. This shunt impedance reduction implies that the cavity fields are smaller than in the cylinder by $7.6 \%$ at the same power level. The main penalty for using the vaned cavity in place of the cylinder is the increase by $260 \%$ in the ratio of peak surface electric field to electric field at the location of the beam $\left(r \approx r_{o}=65 \mathrm{~cm}\right)$. However, since the peak surface fields for the cylindrical case are already relatively low $(3.8 \mathrm{MV} / \mathrm{m}$ in the $8^{\text {th }}$ cavity), this enhancement factor is probably not significant.

Table III. Parameters for dipole mode in $8^{\text {th }}$ cavity, ideal case and with radial vanes.

\begin{tabular}{|c||c|c|}
\hline & ideal cylindrical cavity & cavity with 4 vanes \\
\hline \hline dipole mode resonance frequency, $\mathrm{MHz}$ & 64.0 & 64.0 \\
\hline aperture diameter, cm & 344 & 150 \\
\hline outer cavity diameter, cm & 344 & 240 \\
\hline cavity length, cm & 390 & 390 \\
\hline quality factor $Q_{O}$ & 145,000 & 42,500 \\
\hline transverse shunt impedance $Z_{\perp}, \mathrm{M} \Omega / \mathrm{m}^{2}$ & 4.1 & 3.5 \\
\hline$Z_{\perp} / Q_{O}, \Omega / \mathrm{m}^{2}$ & 28 & 84 \\
\hline$E_{\text {Surf, } \max } / E(65 \mathrm{~cm})$ & 0.96 & 2.5 \\
\hline
\end{tabular}

*For these considerations, the appropriate cavity figure-of-merit for comparing different cavity designs is the transverse shunt impedance, defined as $Z_{\perp}=E_{\perp}^{2}\left(r_{o}\right) / P$ where $P$ is the power dissipated in cavity ohmic losses, i.e. without inclusion of interactions with the beam, and $r_{o}$ is the beam location. This follows since it is the transverse RF electric field that provides proton acceleration. Note that the units for $Z_{\perp}$ are $\mathrm{M} \Omega / \mathrm{m}^{2}$. 


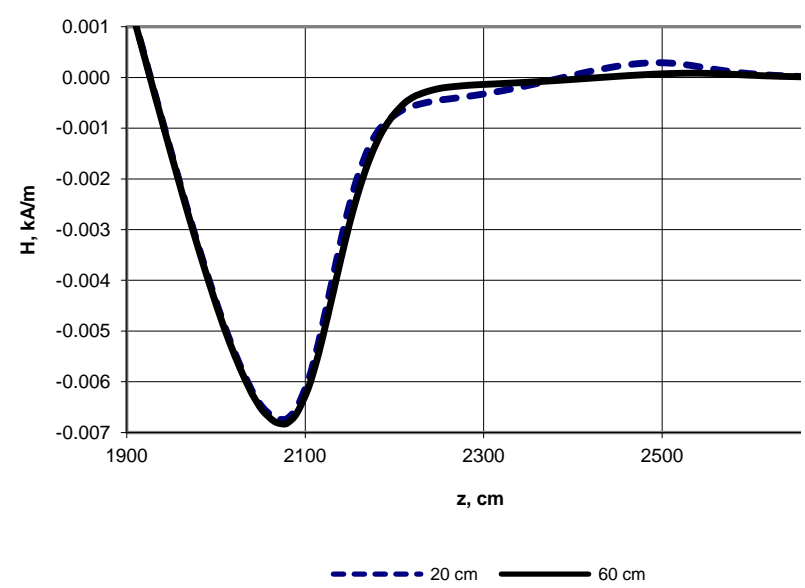

Fig. 8. Longitudinal profile of the transverse magnetic field on axis for the $7^{\text {th }}$ cavity (the worst case). Results are shown for a $20-\mathrm{cm}$ drift tunnel (dashed curve) and a $60-\mathrm{cm}$ drift tunnel (solid curve). The $7^{\text {th }}$ cavity ends at $2120 \mathrm{~cm}$. One can see that even for a $20-\mathrm{cm}$ drift tunnel the field of the $7^{\text {th }}$ cavity that penetrates into the $8^{\text {th }}$ cavity is quite small.

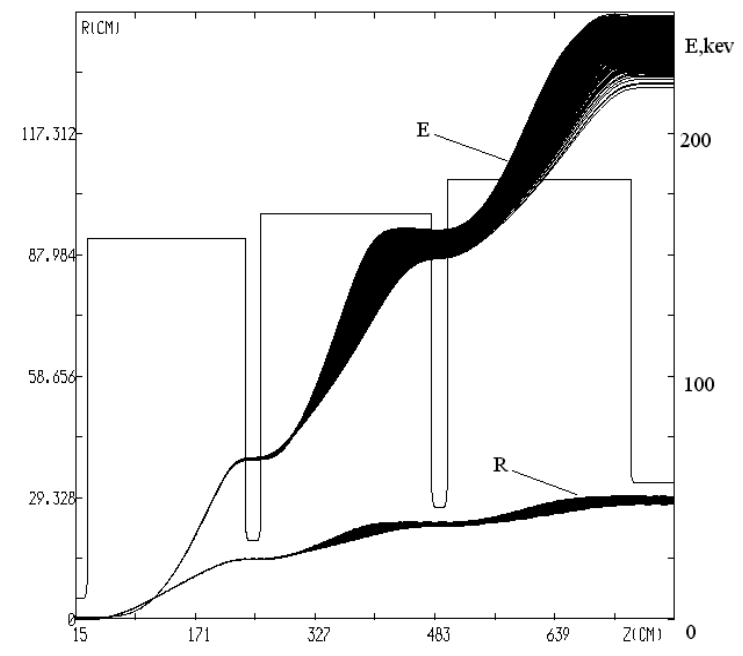

Fig. 10. Energy and radius versus $z$ for protons in a Brillouin beam, with $I=915 \mathrm{~mA}$ and duty $=1 / 7.5$. Cavity and drift tunnel outlines are shown to scale. Note close similarity to Fig. 9.

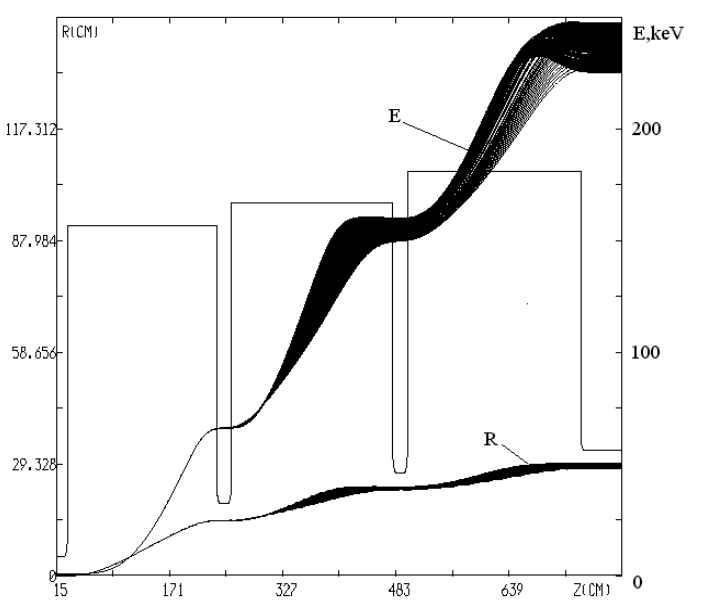

Fig. 9. Energy and radius versus $z$ for accelerated protons in a cold beam. $I=915 \mathrm{~mA}$ and duty $=1 / 7.5$. Cavity and drift tunnel outlines are shown to scale.

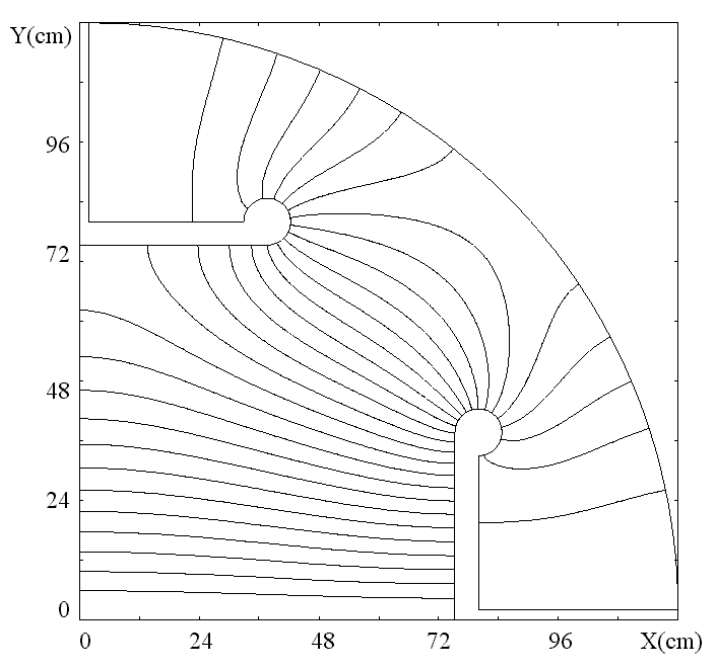

Fig. 11. Structure transverse outline and dipole electric field pattern, for vaned cavity (one quadrant only). Degenerate orthogonal dipole mode also to be excited has vertical field lines near the center. 


\section{Omega-P, Inc.}

Final Report, DoE SBIR Grant DE-FG02-07 ER 84862

HIGH-INTENSITY PROTON ACCELERATOR

\section{TECHNICAL APPROACH - CONCEPTUAL DESIGN FOR A COUNTERPART MULTI-CAVITY ELECTRON CYCLOTRON}

Since it is plainly unrealistic to design, build, and test even a portion of the multicavity proton cyclotron described above within the time and cost constraints of an SBIR project, it was decided that an electron counterpart model be developed, for construction and testing during Phase II. This is to allow laboratory confirmation of the basic multifrequency acceleration mechanism, including means for adjustment of the injection phase window in the first cavity from narrow to wide, and means for adjustment of the beam loading of the cavities from light to heavy. The main parameters of the electron counterpart experiment have been chosen to closely mimic corresponding parameters for a proton machine. Thus, the goal for the electron counterpart is to produce continuous multi-frequency acceleration to $\sim 511 \mathrm{keV}(\gamma=2)$, since the proton machine was modeled to accelerate to about $\sim 938 \mathrm{MeV}(\gamma=2)$. Injected beam velocities are similar, with a $1 \mathrm{keV}$ electron beam $(\beta=0.063)$, as compared with $1000 \mathrm{keV}$ for the protons $(\beta=$ $0.046)$. The main exceptions to parameter similarity are in the RF powers and magnetic fields. For the proton case, average RF and beam powers are 174 and $116 \mathrm{MW}$; while for the $8-\mathrm{mA}$ electron case these values are 5.8 and $4.1 \mathrm{~kW}$. Axial magnetic field for the electron example is $0.155 \mathrm{~T}$, compared with $8.1 \mathrm{~T}$ for the proton case. With these parameters for an electron counterpart, underlying physics of multi-frequency cyclotron acceleration can be examined under conditions not dissimilar from the proton case.

Simulations were carried out for a preliminary version of a four-cavity electron counterpart, rather than eight cavities as used for the proton case discussed above. This reduction in numbers of cavities is possible since the required power levels for each cavity in the electron accelerator will introduce insignificant cavity wall heat loading, whereas accommodating a level of about $15 \mathrm{MW}$ to be dissipated on each of four cavities in a proton machine would be a challenge. Moreover, reduction in length in the proton machine would probably lead to lower acceleration efficiency and need for even greater $\mathrm{RF}$ drive power, for a fixed beam power. Furthermore, for the electron counterpart, the simplest design consistent with demonstration of the essential physics was desired. Parameters are shown in Table VI for a four-cavity electron cyclotron $8 \mathrm{~mA}$ average current and $1.0 \mathrm{keV}$ injected beam, corresponding to relatively heavy beam loading. The perveance for this injected beam is $0.25 \times 10^{-6} \mathrm{~A}-\mathrm{V}^{-3 / 2}$.

Table IV. Parameters for 4-cavity electron counterpart cyclotron. $I=8 \mathrm{~mA}$, duty $=\mathbf{1} / \mathbf{1 0}$.

\begin{tabular}{|c||c|c|c|c|c|c|c|c|}
\hline $\begin{array}{c}\text { stage } \\
\#\end{array}$ & $\begin{array}{c}\text { cavity } \\
\text { freq. } \\
(\mathrm{GHz})\end{array}$ & $\begin{array}{c}\text { cavity } \\
\text { radius } \\
(\mathrm{cm})\end{array}$ & $\begin{array}{c}\text { cavity } \\
\text { length } \\
(\mathrm{cm})\end{array}$ & $\begin{array}{c}\text { rf } \\
\text { power } \\
\text { input } \\
(\mathrm{kW})\end{array}$ & $\begin{array}{c}\text { relative } \\
\text { rf } \\
\text { phase }\end{array}$ & $\begin{array}{c}\text { beam- } \\
\text { loaded } \\
\text { cavity } \\
Q_{\mathrm{L}}\end{array}$ & $\begin{array}{c}\text { intrinsic } \\
\text { cavity } Q_{\mathrm{o}} \\
\text { (copper) }\end{array}$ & $\begin{array}{c}\text { mean } \\
\text { energy } \\
\text { gain } \\
\text { (keV) }\end{array}$ \\
\hline \hline 1 & 4.00 & 2.75 & 6.23 & 1.08 & 0 & $3.51 \times 10^{3}$ & $1.80 \times 10^{4}$ & 108.4 \\
\hline 2 & 3.50 & 3.15 & 7.09 & 1.00 & $0.57 \pi$ & $3.94 \times 10^{3}$ & $2.00 \times 10^{4}$ & 100.4 \\
\hline 3 & 3.00 & 3.65 & 8.37 & 1.71 & $1.03 \pi$ & $6.66 \times 10^{3}$ & $2.10 \times 10^{4}$ & 145.6 \\
\hline 4 & 2.50 & 4.40 & 9.96 & 2.00 & $1.38 \pi$ & $8.30 \times 10^{3}$ & $2.30 \times 10^{4}$ & 159.2 \\
\hline \hline total & & & 31.65 & 5.79 & & & & 513.6 \\
\hline
\end{tabular}




\section{Omega-P, Inc.}

Final Report, DoE SBIR Grant DE-FG02-07 ER 84862

HIGH-INTENSITY PROTON ACCELERATOR

For this $8 \mathrm{~mA}$ example, the RF-to-beam efficiency is $71.0 \%$. Cavity frequency separations are $500 \mathrm{MHz}$ and the duty factor is $10 \%$, corresponding to a $0.2 \mathrm{~ns}$ electron pulse injected every $2 \mathrm{~ns}$. [A $500 \mathrm{MHz}$ buncher/injector could be contemplated.] The injection phase window here is $288^{\circ}$ in the first $(4 \mathrm{GHz})$ cavity. [Simulations show that, the greater the number of cavities for a given acceleration energy, the larger can be the injection phase angle but the greater will be the accelerator length.] Fig. 12 shows the computed average electron energy and normalized average axial velocity along the structure; Fig. 13 shows the transverse $x-y$ projection of a typical electron orbit during acceleration through all four cavities; Fig. 14 shows the evolution of spreads in electron energy and axial velocity; Fig. 15 shows energy gain for 16 electrons, injected uniformly throughout the $288^{\circ}$ injection phase window; and Fig. 16 shows a projection of a typical electron orbit in the $x-z$ plane, together with an outline sketch of the cavities. These results are similar to those shown for protons in Figs. 2-5. Of course, acceleration of 8 $\mathrm{mA}$ electron beams to $515 \mathrm{keV}$ in a 32-cm long RF system will set no records. But the objective of the electron counterpart experiments is not the achievement of high acceleration gradient, but rather a demonstration of multi-frequency acceleration with a large phase acceptance in the first cavity, and with adjustable beam loading of the cavities. The experimental set-up is meant to allow for wide adjustments in beam current (thus beam loading) and in injection phase window, to test the limits of this acceleration mechanism.

The recitation in Section II describes, using results of detailed computations, the concept of the multi-cavity proton accelerator. One preliminary conceptual design for a model electron counterpart accelerator has been described in this Section. Details of the electron counterpart being built will be described in Section IV.

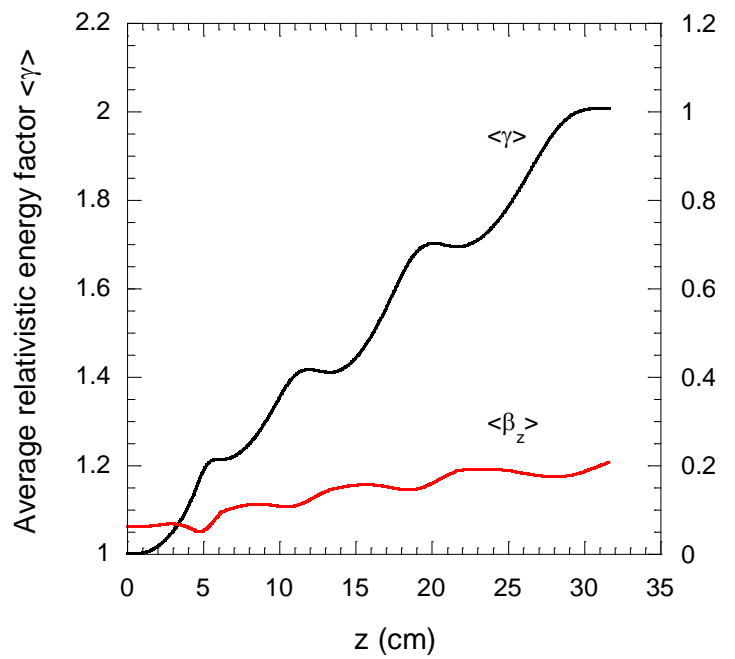

Fig. 12. Dependence of average relativistic energy factor and normalized axial velocity on axial distance for the 4-cavity electron counterpart accelerator.

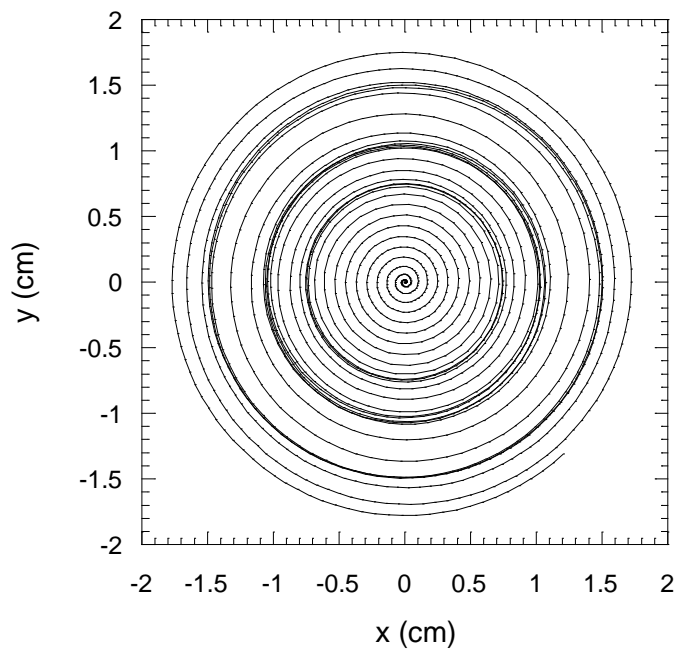

Fig. 13. Projection onto the transverse plane of the orbit of a typical electron. 


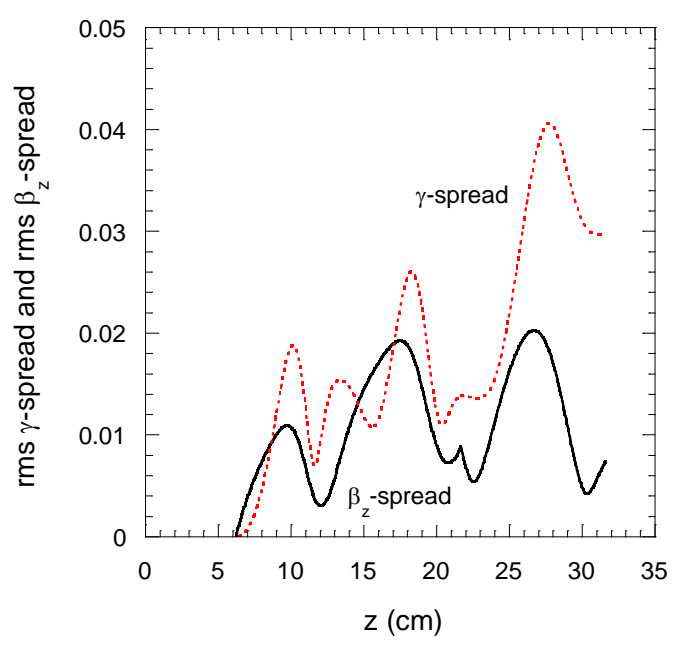

Fig. 14. Dependence of rms spreads in relativistic energy factor and normalized axial velocity, as functions of axial distance $z$. These spreads are seen to be $15 \mathrm{keV}$ and $0.008 c$.

Oscillations with $z$ in these parameters are characteristic of stable orbits.

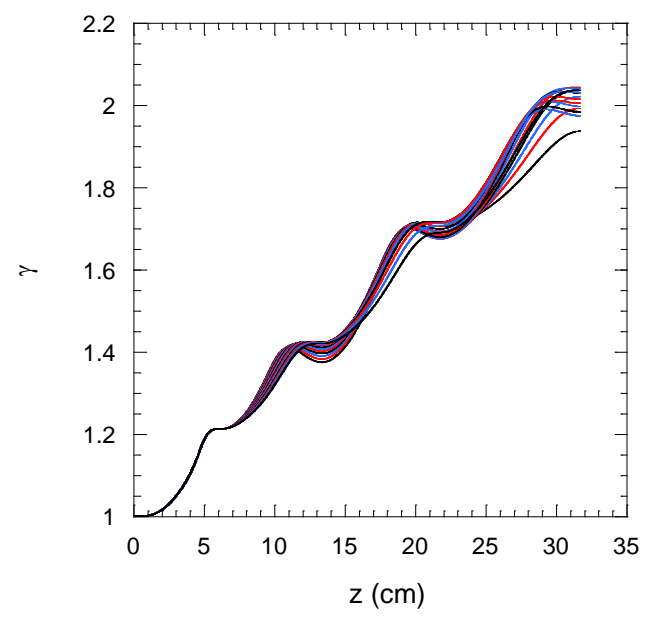

Fig. 15. Dependence of relativistic energy factor on axial distance for 16 particles, distributed uniformly over a $288^{\circ}$ injection phase window in the first cavity, corresponding to a duty factor of $10 \%$.

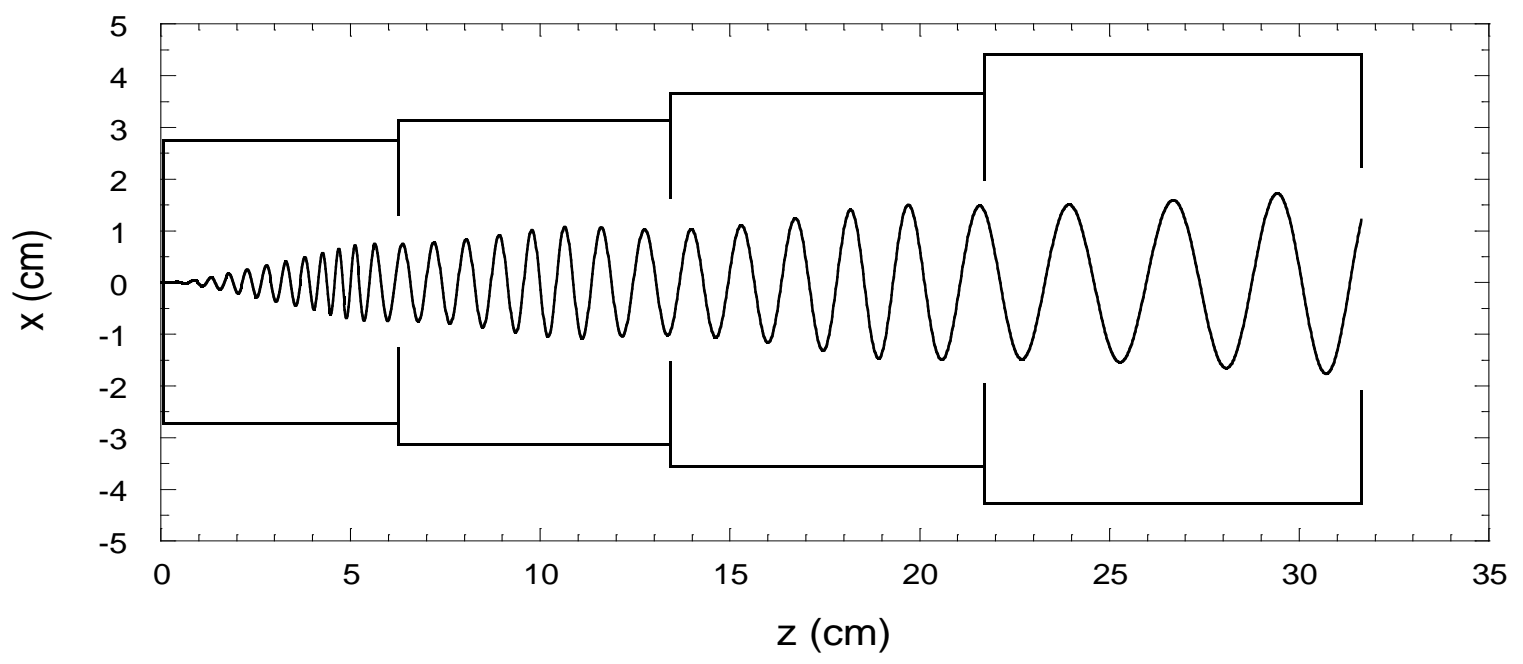

Fig. 16. Projection onto the $x$-z plane of the orbit of a typical particle for the proposed 2.5-4.0 GHz four-cavity electron counterpart cyclotron. Electrons are accelerated from $1 \mathrm{keV}$ to $515 \mathrm{keV}$ with parameters as in Table IV. Outlines of cavities are shown drawn roughly to scale. 


\section{ENGINEERING DESIGN AND PRELIMINARY TESTS FOR COUNTERPART MULTI-CAVITY ELECTRON CYCLOTRON ACCELERATOR}

Experimental work on this project was carried out under a sub-grant from Omega-P to the Beam Physics Laboratory (BPL) at Yale University, with Dr. Michael A. LaPointe serving as Principal Investigator for Yale. A SolidWorks image of the experimental layout of a four-cavity counterpart multi-cavity electron cyclotron accelerator is shown in Fig. 17. The image shows the electron gun injector on the left, with two pumping ports; the four $\mathrm{TE}_{111}$ cavities with equal decreasing frequency increments to match decreasing gyrofrequency with increasing electron energy; and the beam collector at the right. The first three cavities each have two criticallycoupled radial RF feeds phased in quadrature to launch rotating modes, and two weakly-coupled sampling ports. The last cavity has its four RF ports with loop antennas on the end: two for excitation, two for sampling.

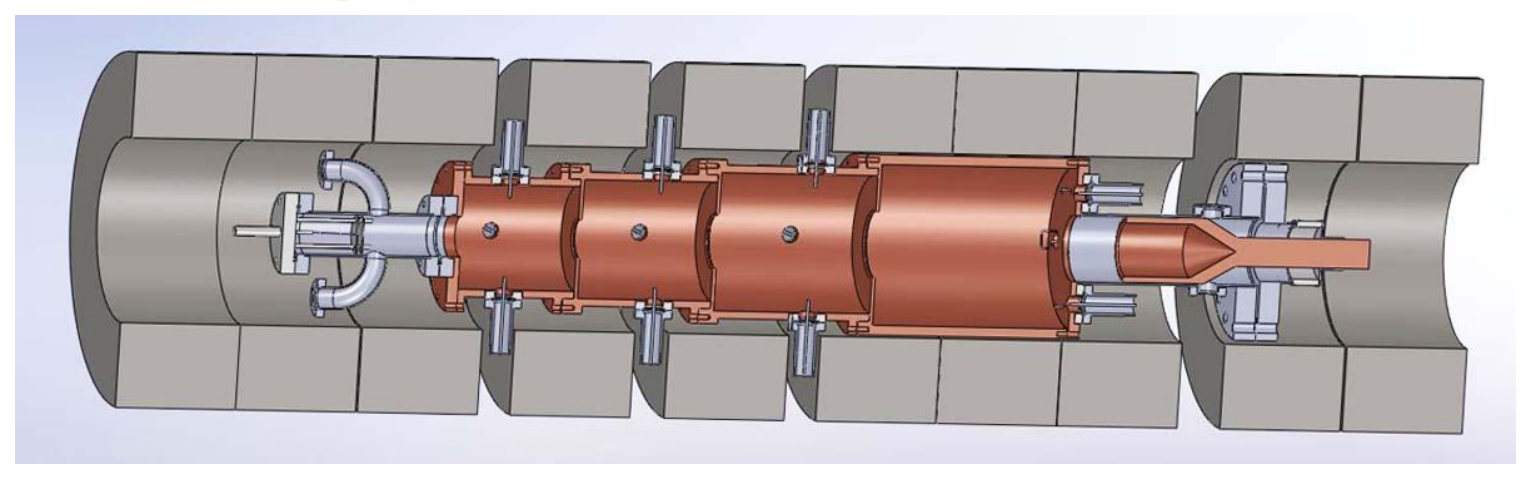

Fig. 17. Layout for the four-cavity electron cyclotron accelerator.

The entire structure must be assembled within coils that provide the necessary axial magnetic field. Coils and associated power supplies available at Yale have parameters that limit the design of the cavity system; in particular the coil's inner diameter of $15 \mathrm{~cm}$ limits the maximum diameter of the lowest frequency cavity - and thus strongly dictates the lowest frequency itself, while the highest peak magnetic field of about $2 \mathrm{kG}$ that can be sustained sets an upper limit for the highest frequency of the highest frequency cavity. Balancing these competing factors led to the compromise as follows: magnetic field strength $B \approx 1.6 \mathrm{kG}$, and cavity frequencies of 2.4 , 2.1, 1.8, and $1.5 \mathrm{GHz}$. A photo of the coil system is shown in Fig. 18, with measurements of the axial field profile shown in Fig. 19. Each coil is powered from its own dedicated power supply.

The cavity system is of necessity of demountable design. This is to permit tuning of cavities individually by machining radii and lengths as needed, and to allow convenient access ports for four coaxial feed and sampling antennas in each cavity, without need for multiple iterations in fabrication and associated excessive costs that would attend use of a one-piece brazed assembly. As a consequence, the unloaded cavity $Q$ factors were lower than for a design without demountable joints. This in turn led to a decision not to operate the first prototype of the accelerator in a range where beam loading would further lower the $Q$ factors, as this would entail need for higher levels of RF drive power to achieve a given acceleration. Thus, rather than select an injector gun with a current in the 5-10 mA range, a design for the 10-50 $\mu \mathrm{A}$ range was chosen. This would of course prevent measurements of the ultimate efficiency of the accelerator, but would permit determination of the less-understood phase acceptance of the multi-cavity 


\section{Omega-P, Inc.}

Final Report, DoE SBIR Grant DE-FG02-07 ER 84862

\section{HIGH-INTENSITY PROTON ACCELERATOR}

acceleration mechanism - a phenomenon that has a direct bearing on the efficiency as well as the beam quality.

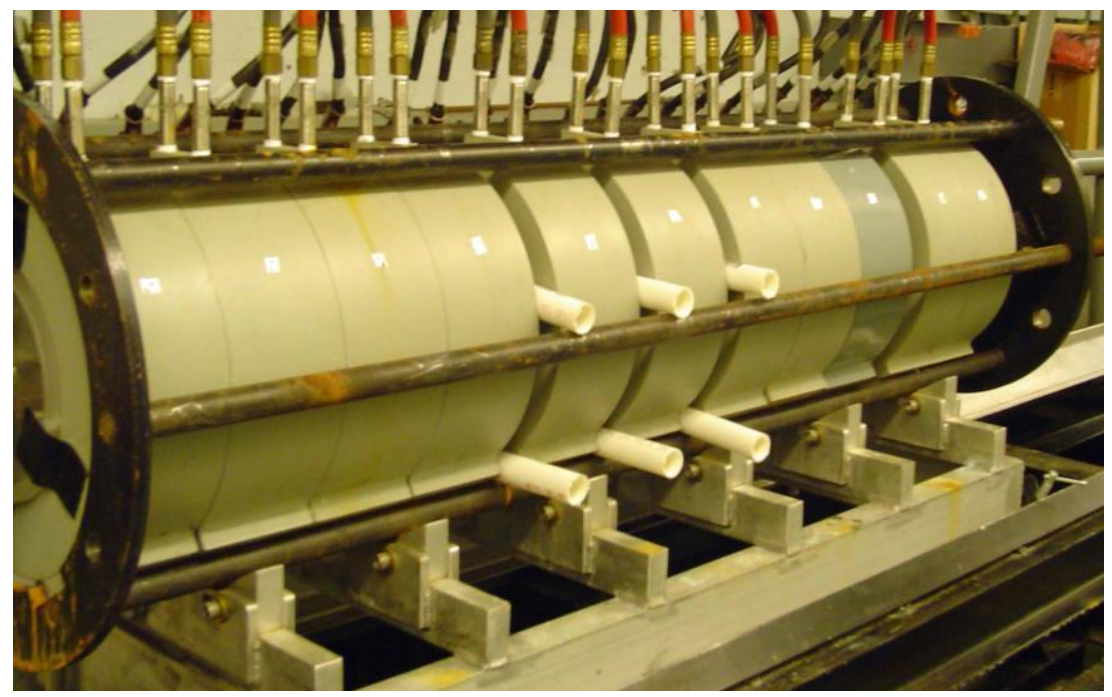

Fig. 18. Coil system for a four-cavity electron cyclotron accelerator. Spacers between coils provide gaps where coaxial RF feed and sampling cables are being placed.

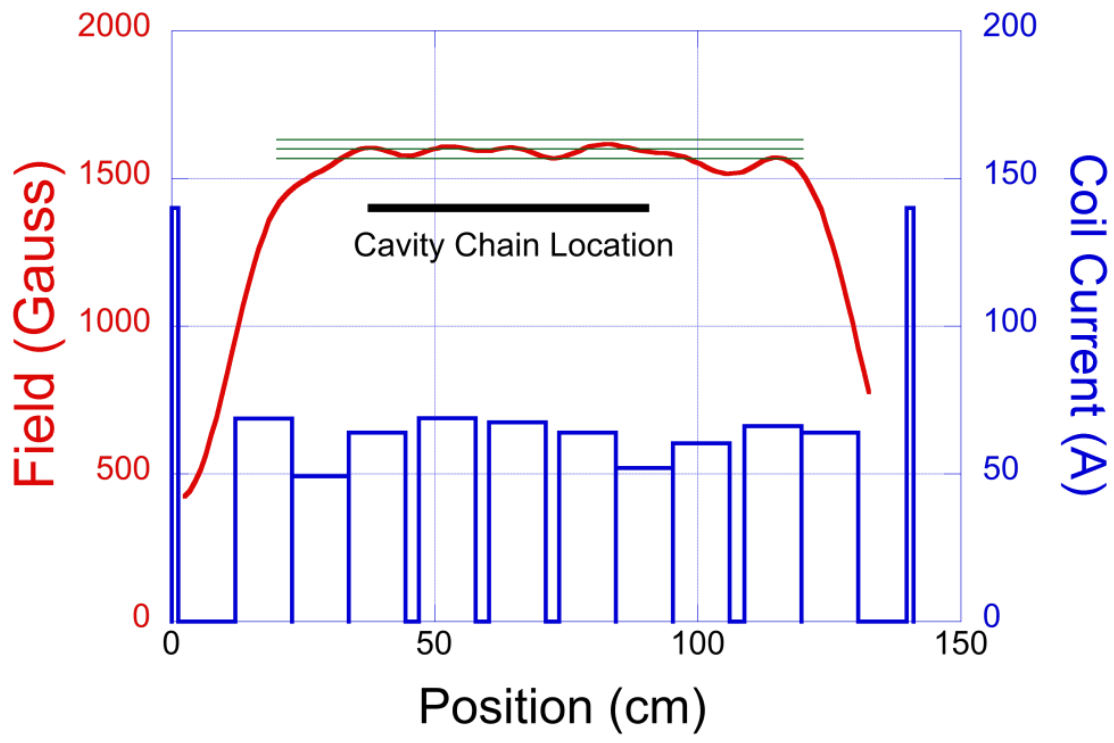

Fig. 19. Measured axial magnetic field along center-line in the coil system, shown in red. Histogram in blue shows currents in individual coils used to achieve this field profile. Iron pole pieces are placed at $0 \mathrm{~cm}$ and at $140 \mathrm{~cm}$. The peak-to-peak field variation along the cavity chain is less than $\pm 2 \%$.

The electron gun design chosen for first experiments is depicted in Fig. 20. It incorporates a Kimball Physics ES-015 BaO cathode in a CB-104 support. The cathode assembly is held in place by a two-piece focusing electrode, seen in Fig. 20. The anodecathode spacing is $5 \mathrm{~mm}$. The cathode operates at $1200 \mathrm{~V}$ with a maximum current of 50 


\section{Omega-P, Inc.}

Final Report, DoE SBIR Grant DE-FG02-07 ER 84862

\section{HIGH-INTENSITY PROTON ACCELERATOR}

$\mu$ amps, corresponding to a perveance of $1.2 \times 10^{-9} \mathrm{~A}-\mathrm{V}^{-3 / 2}$. The gun can be pulsed for up to 10 $\mu \mathrm{sec}$ at a maximum repetition rate of $10 \mathrm{~Hz}$, in synchronism with pulsing of the cavity's RF sources, or as in first experiments, the gun can be operated CW. Photos of the assembled gun are shown in Fig. 21.

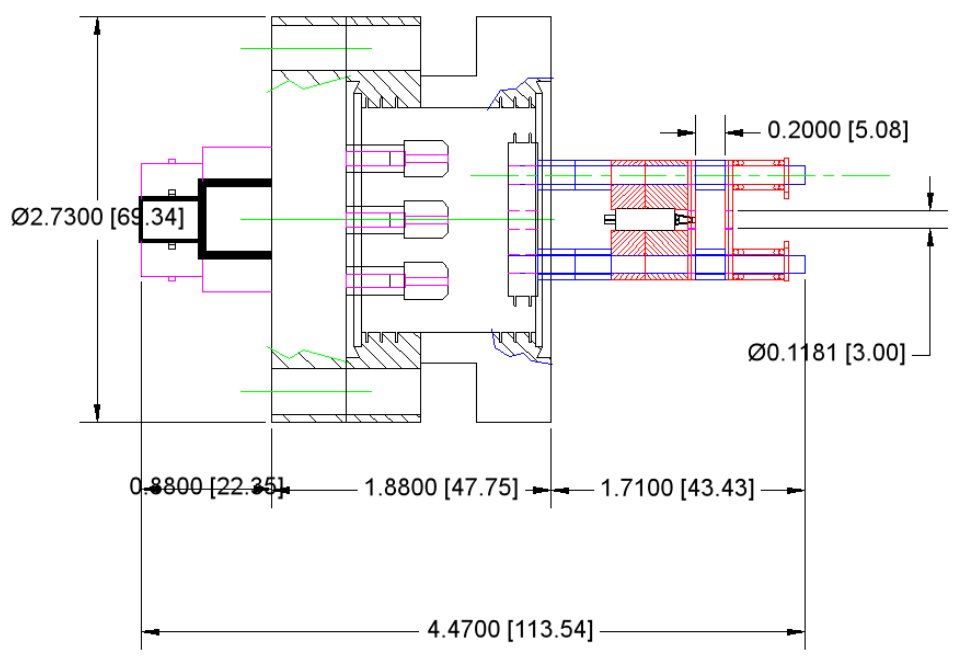

Fig. 20. Electron gun design. Anode and directly-heated cathode are at right side.
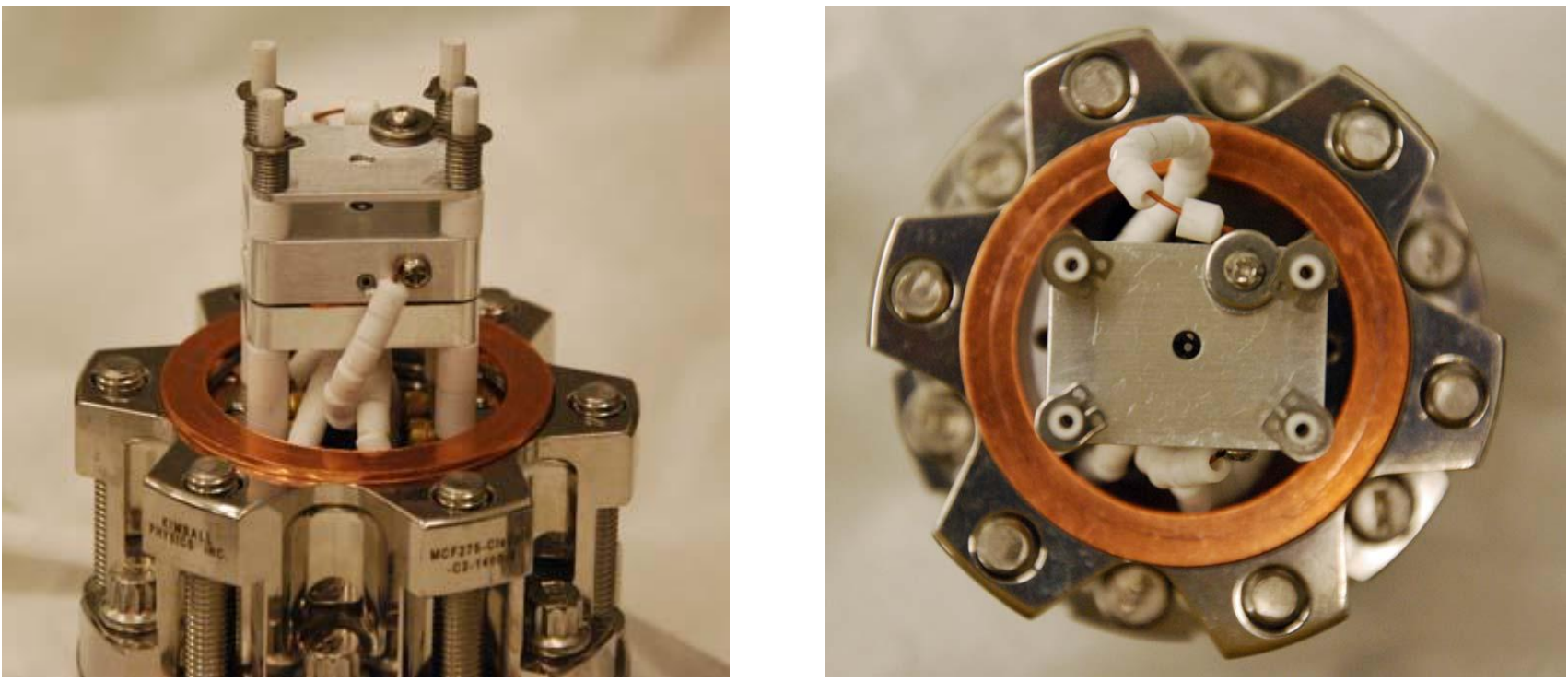

Fig. 21. Photos of the gun assembly.

Cost and availability considerations dictated the choice of the four pulsed RF amplifiers needed for the experimental test of the four-cavity cyclotron accelerator. The units that were 


\section{Omega-P, Inc.}

Final Report, DoE SBIR Grant DE-FG02-07 ER 84862

HIGH-INTENSITY PROTON ACCELERATOR

chosen, supplied by the UK firm Microwave Amplifiers Ltd., are rated to supply $500 \mathrm{~W}$ peak pulses at a duty factor not to exceed 1\%. As stated, the amplifiers operate at 1.5, 1.8, 2.1, and $2.4 \mathrm{GHz}$; they are driven at harmonics of $300 \mathrm{MHz}$ as supplied by a Spectrum Microwave custom-built source, so that all four signals that drive the amplifiers are in strict phase synchrony. The four-frequency RF sources are shown in Figs. 22-24. Fig. 22 shows the 300 $\mathrm{MHz}$ phase-stable master oscillator with the four outputs at its $5^{\text {th }}, 6^{\text {th }}, 7^{\text {th }}$, and $8^{\text {th }}$ harmonics.

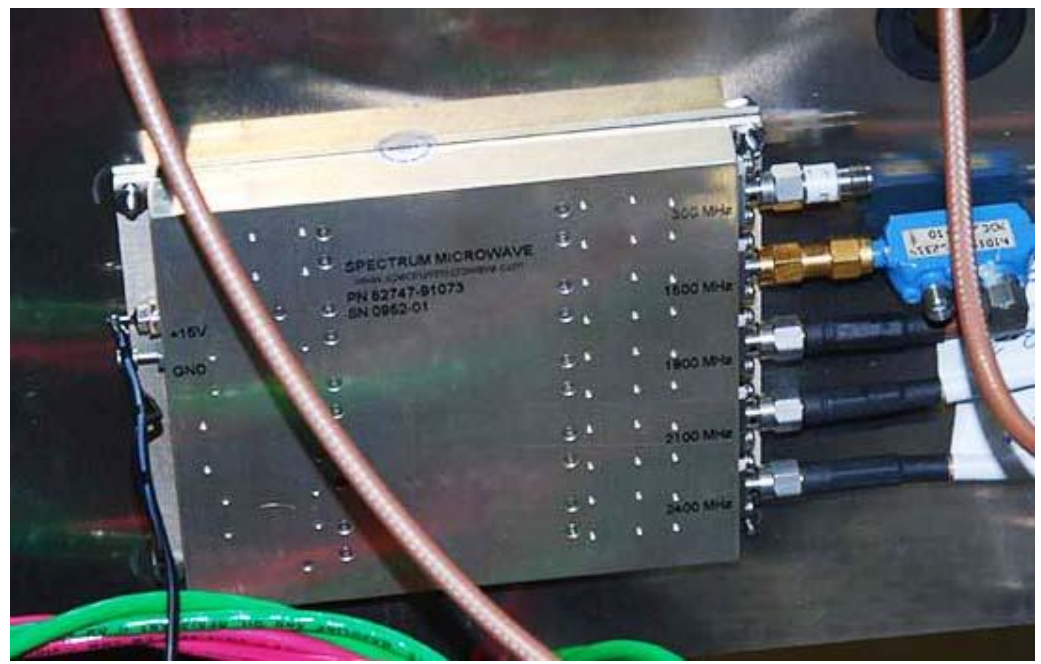

Fig. 22. $300 \mathrm{MHz}$ master phase-locked oscillator with the four outputs at its $5^{\text {th }}, 6^{\text {th }}, 7^{\text {th }}$, and $8^{\text {th }}$ harmonics, namely $1.5,1.8,2.1$, and $2.4 \mathrm{GHz}$

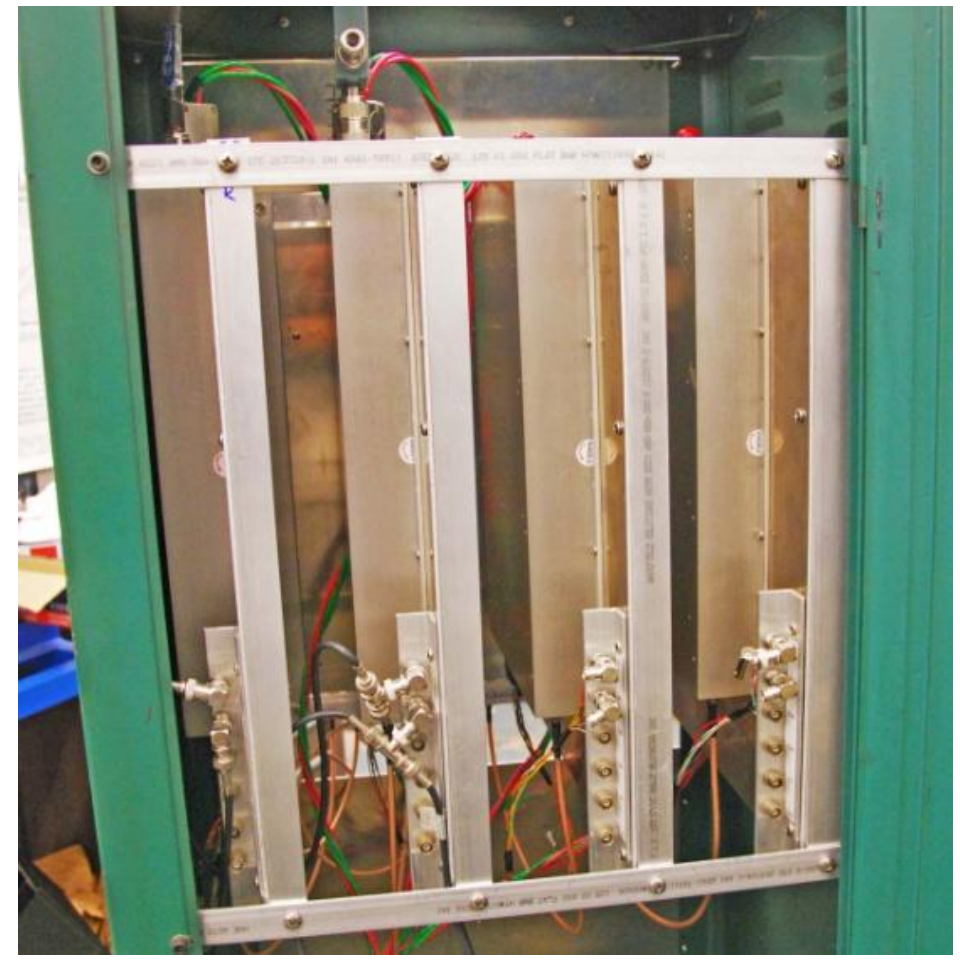

Fig. 23. Four 500-W amplifiers, at 1.5, 1.8, 2.1, and $2.4 \mathrm{GHz}$ as mounted in the RF cabinet. 
Omega- $P$, Inc.

Final Report, DoE SBIR Grant DE-FG02-07 ER 84862

HIGH-INTENSITY PROTON ACCELERATOR

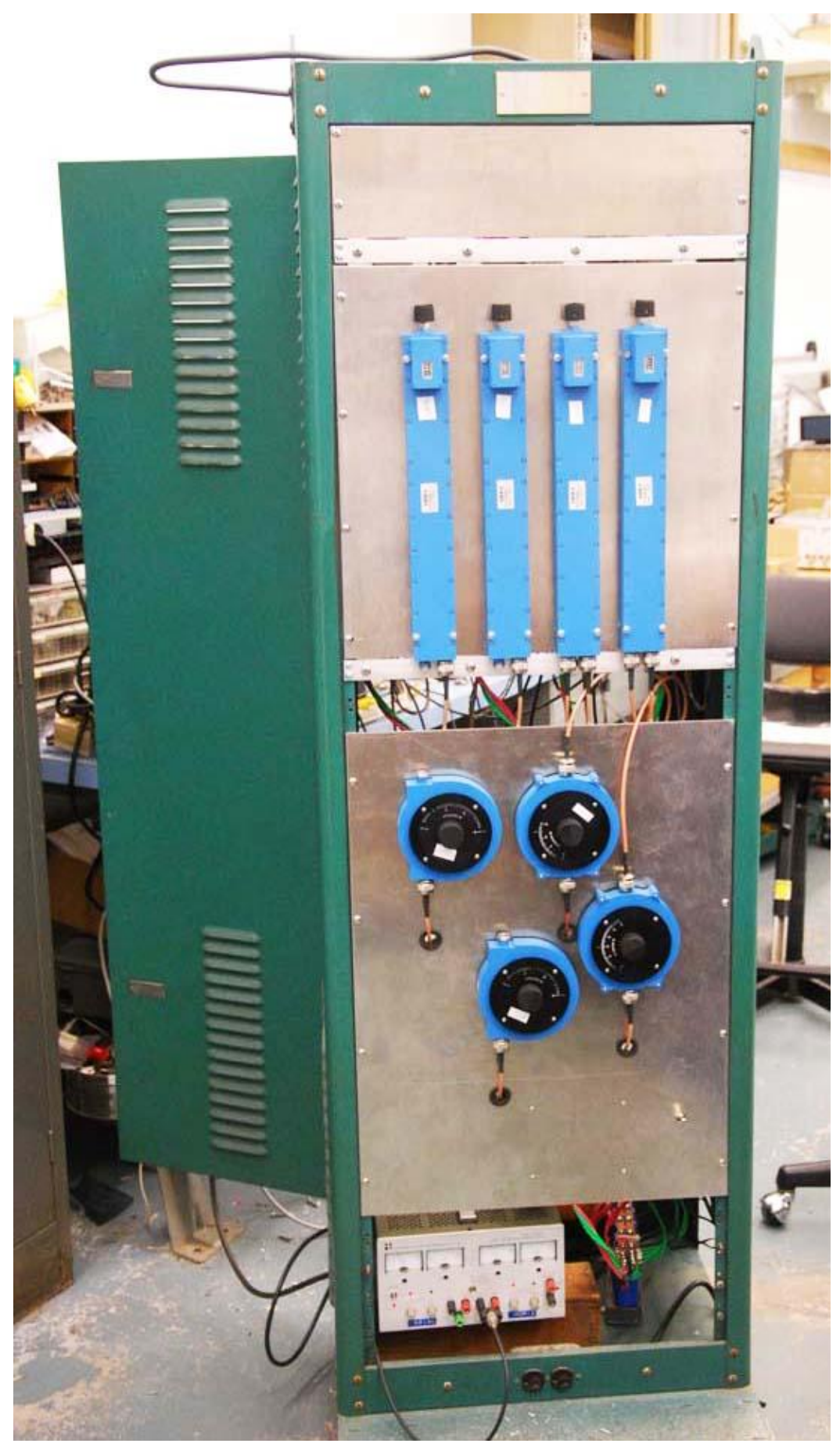

Fig. 24. RF cabinet. Amplifiers are mounted in the rear of the cabinet, as shown in Fig. 23. Shown on front are four precision phase shifters (at top) and four precision attenuators, both needed to adjust drive signals into each amplifier for optimum synchronism and field amplitudes amongst the four cavities. Power supply at bottom charges a capacitor bank that supplies the $\sim 100$ A current surges needed by the amplifiers during each RF pulse. 
Omega- $P$, Inc.

Final Report, DoE SBIR Grant DE-FG02-07 ER 84862

HIGH-INTENSITY PROTON ACCELERATOR

Evidence for good phase control for the $1.5 \mathrm{GHz}$ portion of the system is illustrated in Fig. 25. The dark green trace in the figure is from the $300 \mathrm{MHz}$ master oscillator. The red trace is the sampled $1.8 \mathrm{GHz}$ high power RF signal. The various shades of blue are the sampled $1.5 \mathrm{GHz}$ high power $\mathrm{RF}$ at different phase settings $\left(0^{\circ}\right.$ is dark blue, $90^{\circ}$ is lighter blue, $180^{\circ}$ is lighter blue, and $270^{\circ}$ is lightest blue). All three signals were simultaneously sampled and four data sets were taken, one for each phase. The data were recorded on an Agilent Infinium 54855A digital sampling oscilloscope, while the trigger system was armed by the RF gate signal and then triggered by a positive rising level on the sampled $300 \mathrm{MHz}$ master signal.

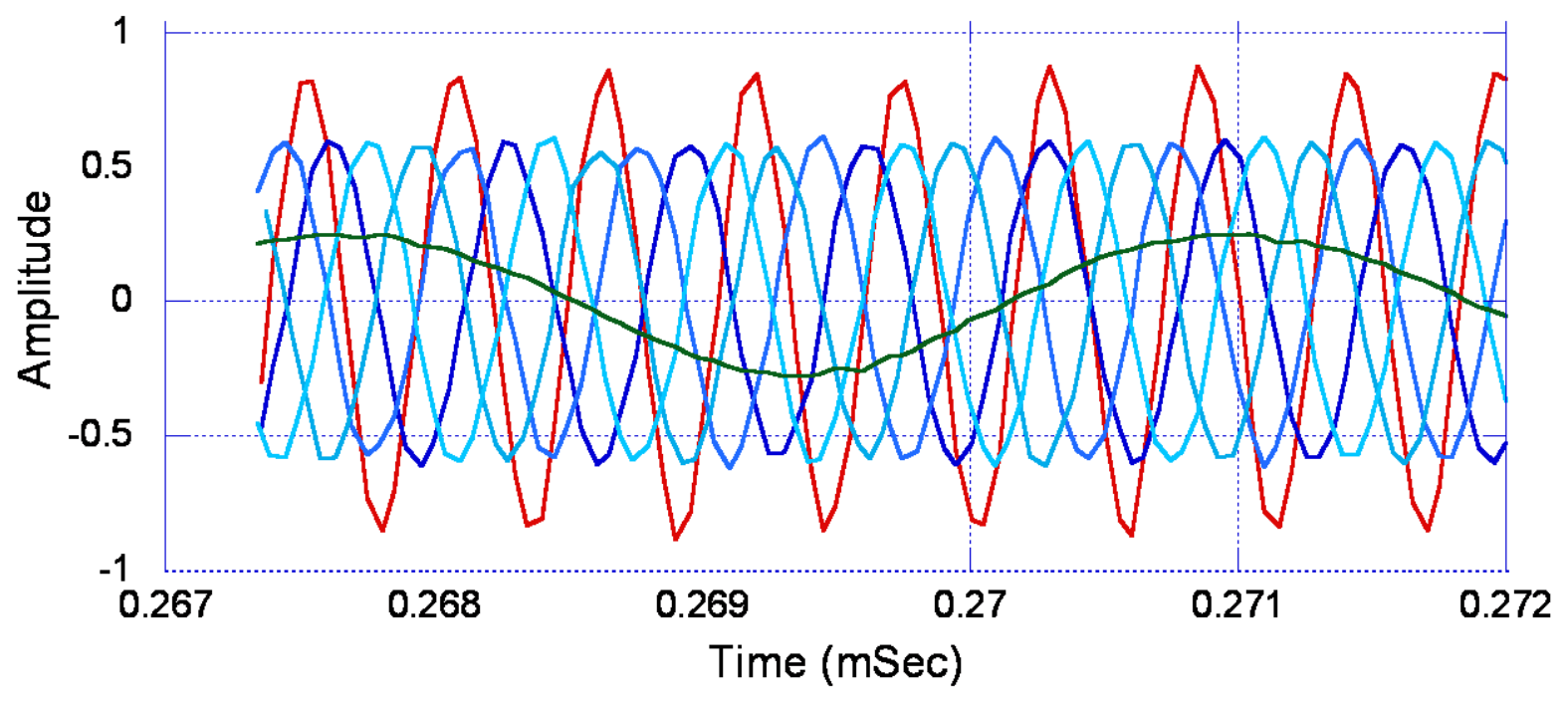

Fig. 25. Demonstration of phase control. See text for explanation.

Computed radius $R$ and length $L$ for each of the four cavities being built for the electron accelerator are listed in the table below. Also listed in the table are the aperture radius $a$ and Ohmic $Q$ values for each cavity, based on use of OHC copper. For these $Q$-values, the table also gives energy gain values $\Delta U$ for the level of RF power $P$ in each cavity. Irises between cavities are $3 \mathrm{~mm}$ thick. Final cavity tuning will either increase $R$ or decrease $L$ to lower or raise the actual mode frequencies to match the design frequencies of 1500, 1800, 2100, and $2400 \mathrm{MHz}$.

\begin{tabular}{|c||c|c|c|c|c|c|c|}
\hline $\begin{array}{c}\text { cavity } \\
\#\end{array}$ & $\begin{array}{c}f_{\text {TE111 }} \\
\mathrm{GHz}\end{array}$ & $\begin{array}{c}R \\
\mathrm{~mm}\end{array}$ & $\begin{array}{c}L \\
\mathrm{~mm}\end{array}$ & $\begin{array}{c}a \\
\mathrm{~mm}\end{array}$ & $Q_{\text {ohmic }}$ & $\begin{array}{c}P \\
\text { Watts }\end{array}$ & $\begin{array}{c}\Delta U \\
\mathrm{keV}\end{array}$ \\
\hline \hline 1 & 2.4 & 45.37 & 104 & 16.3 & 24300 & 207 & 119 \\
\hline 2 & 2.1 & 51.21 & 119 & 22.8 & 26000 & 420 & 123 \\
\hline 3 & 1.8 & 59.24 & 139 & 28.4 & 28500 & 367 & 105 \\
\hline 4 & 1.5 & 70.05 & 174 & 33.6 & 30800 & 426 & 147 \\
\hline \hline total & & & 536 & & & 1420 & 494 \\
\hline
\end{tabular}

Field maps showing contours of constant RF magnetic field are shown in Fig. 26. 
Omega-P, Inc.

Final Report, DoE SBIR Grant DE-FG02-07 ER 84862

HIGH-INTENSITY PROTON ACCELERATOR
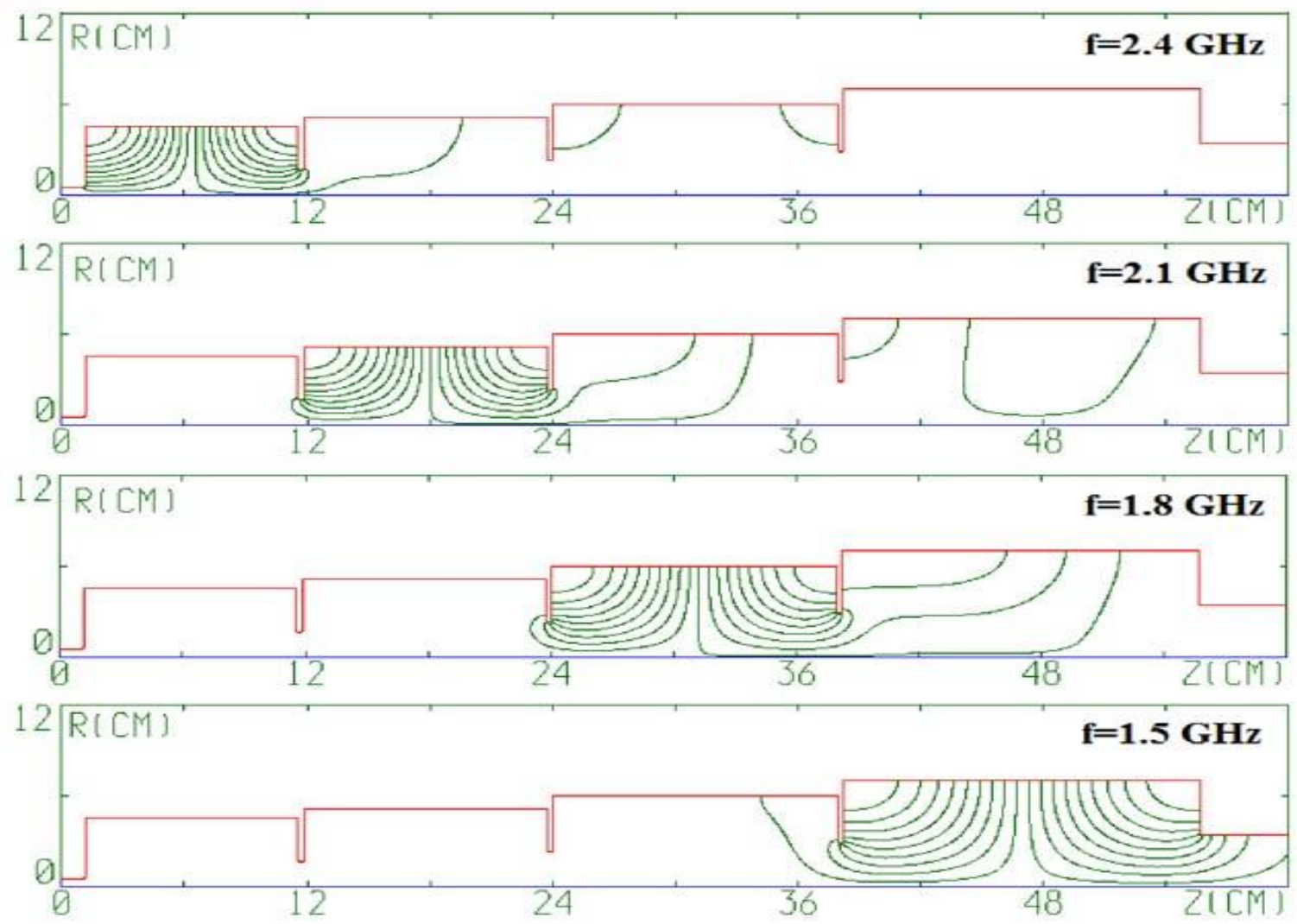

Fig. 26. Field maps at each resonance frequency in the four-cavity structure.

An engineering drawing of the demountable four-cavity structure is shown in Fig. 27. The electron gun injector is joined at the left, while the beam collector and beam energy analyzer is joined at the right. The first three cavities $(2400,2100$, and $1800 \mathrm{MHz})$ have four radial antennas, two for input coupling of up to $500 \mathrm{~W}$ per cavity, and two for diagnostics. The final $1500 \mathrm{MHz}$ cavity has its four coupling loop antennas inserted through the end plate, as necessary because of limited radial access due to the $15-\mathrm{cm}$ inner diameter of the magnet coils.

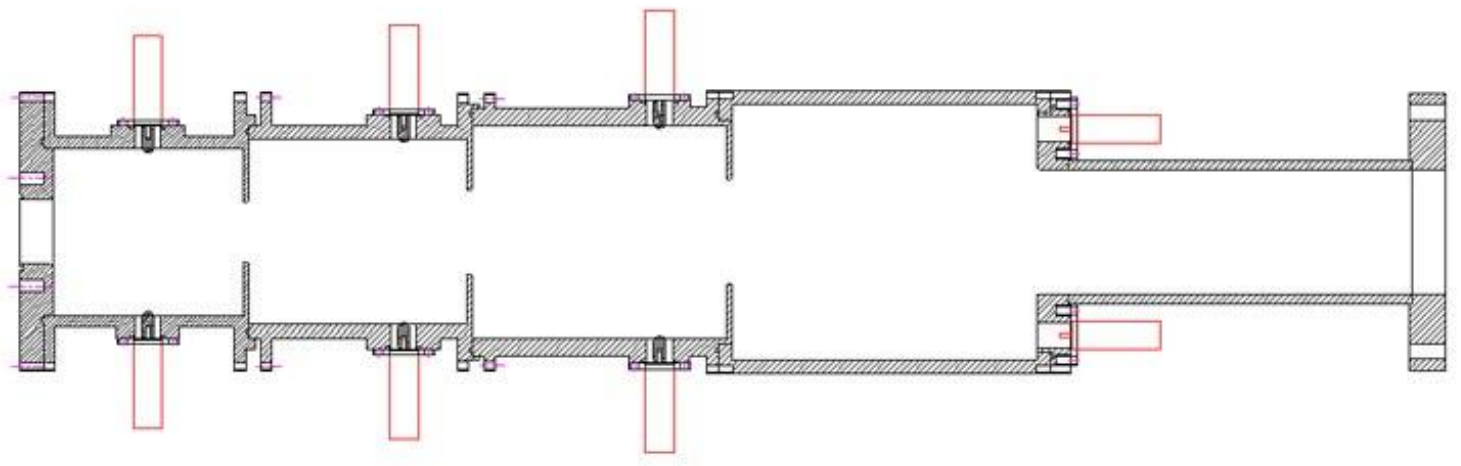

Fig. 27. Engineering outline drawing of the four-cavity electron counterpart accelerator. Critical dimensions for each cavity are given in the table on p. 21. 


\section{Omega-P, Inc.}

Final Report, DoE SBIR Grant DE-FG02-07 ER 84862

HIGH-INTENSITY PROTON ACCELERATOR

Photos of the $2.4 \mathrm{GHz}$ cavity under cold test are shown in Figs. 28 and 29. The former shows the aperture between this cavity and the next, while the latter shows the cavity joined to the electron gun at one end and the beam collector at the other. This latter arrangement is to be used for first tests of beam acceleration in a single cavity, a test designed to allow evaluation of $\mathrm{RF}$ and beam diagnostics.

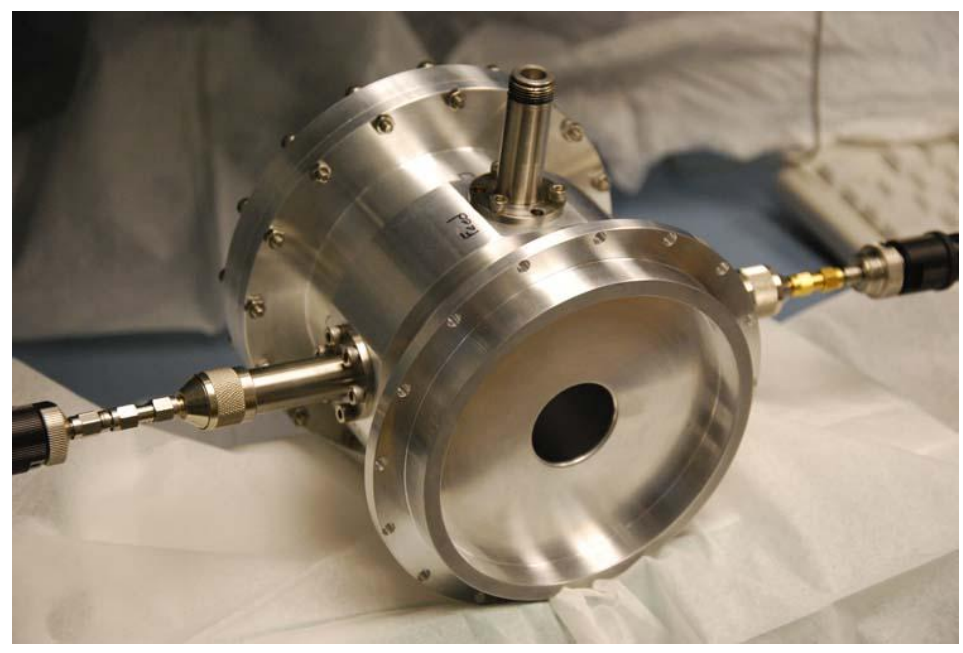

Fig. 28. Photo of $2.4 \mathrm{GHz}$ cavity under cold test, with drive antenna on left and sampling antenna on right. Quadrature mode is to be driven from top location with a $90^{\circ}$ phase lag or lead with respect to the first mode, to effect a clockwise or counter-clockwise rotation of the fields.

Sampling antenna for the quadrature mode is at bottom of cavity.

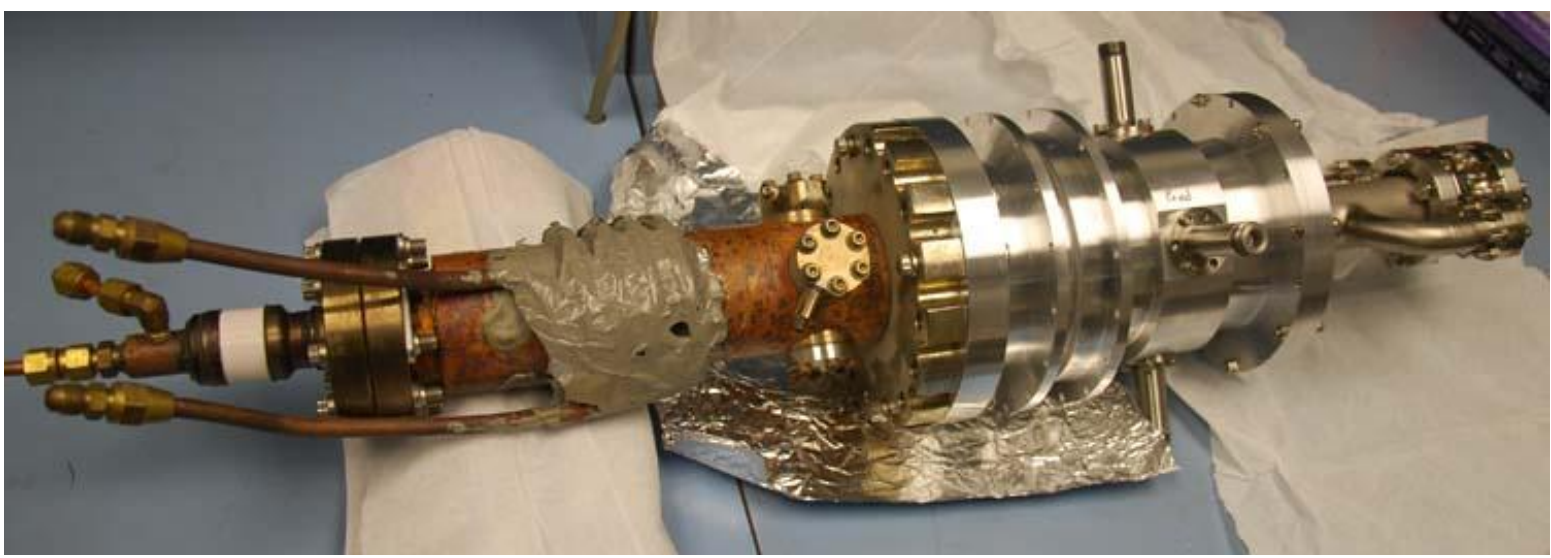

Fig. 29. Photo of $2.4 \mathrm{GHz}$ cavity, assembled with electron gun (at right) and beam collector (at left). This assembly was used for the S-parameter data shown in Fig. 30. 
Omega-P, Inc.

Final Report, DoE SBIR Grant DE-FG02-07 ER 84862

HIGH-INTENSITY PROTON ACCELERATOR

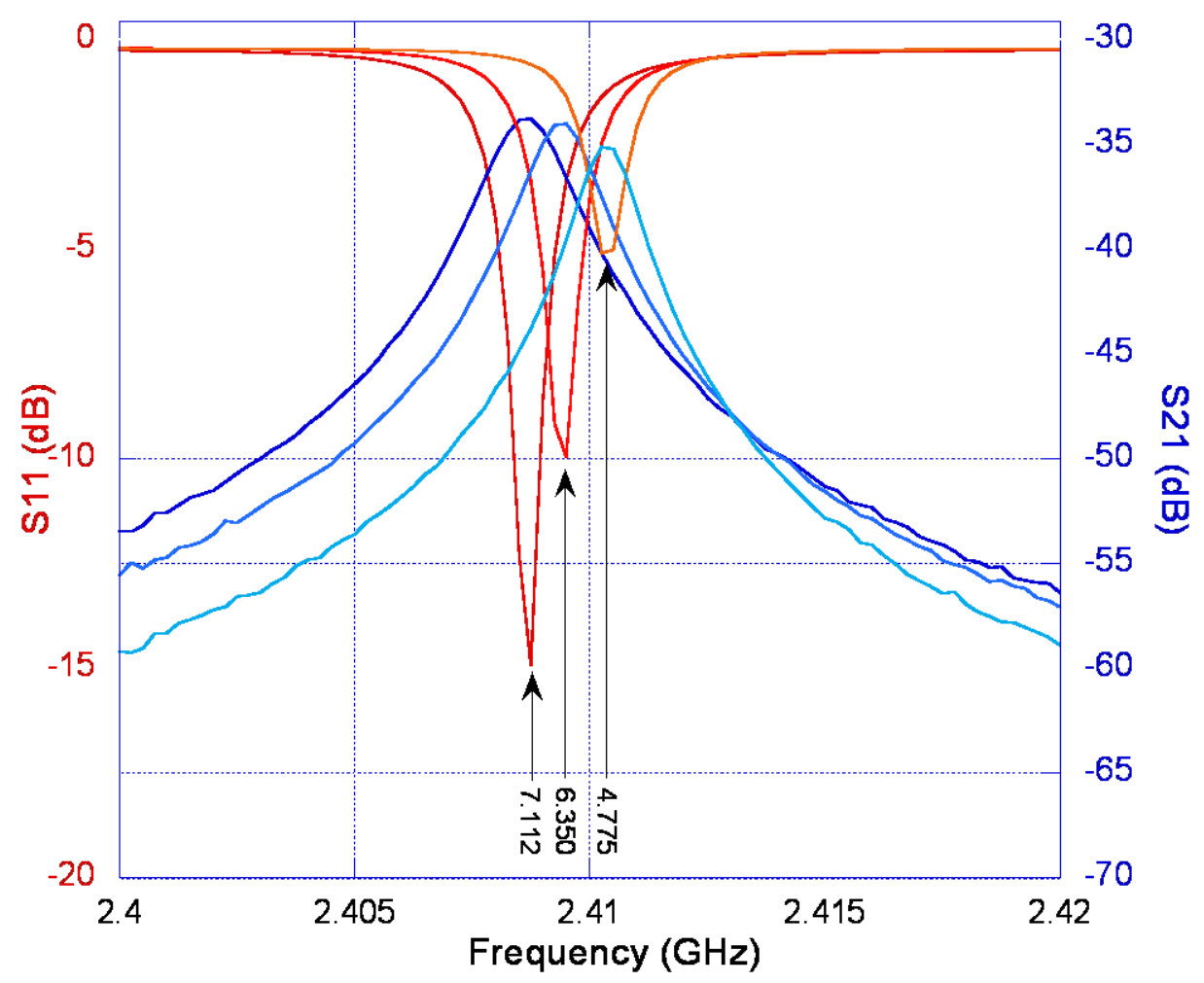

Fig. 30. S-parameter results for one of the two $\mathrm{TE}_{111}$ cavity modes for the assemblied cavity as shown in Fig. 30 for the three indicated values of drive antenna insertion, as measured using an Agilent PNA Model E8364C vector network analyzer.

Data obtained from the scans shown in Fig. 30 are summarized in the table below, showing increased coupling, frequency pulling, and lowered $Q$-values as the coupling antenna is inserted further into the cavity. With the greatest insertion of $7.62 \mathrm{~mm}$, the measured $\mathrm{S}_{11}$ value indicates that about $3 \%$ of the incident power is reflected, that the mode frequency is about 8.5 $\mathrm{MHz}$ higher than desired, and that the loaded $Q$ is only about 1300 . Data for the second $\mathrm{TE}_{111}$ mode are similar, except for a resonance frequency difference of about $0.25 \mathrm{MHz}$ - probably caused by a small difference in antenna lengths. Coupling between the two $\mathrm{TE}_{111}$ modes is measured to be $-19 \mathrm{~dB}$. These measurements show that the drive antenna needs to be slightly re-designed to reduce the reflected power to $<1 \%$ of the incident, and that the cavity radius needs to be slightly increased to lower the mode's eigenfrequency by about $8 \mathrm{MHz}$.

Table. Measured cavity parameters.

\begin{tabular}{|c|c|c|c|c|}
\hline $\begin{array}{c}\text { Antenna distance } \\
\text { from wall }(\mathrm{mm})\end{array}$ & $\begin{array}{c}\text { Frequency } \\
(\mathrm{GHz})\end{array}$ & S11 $(\mathrm{dB})$ & S21 (dB) & $\mathrm{Q}$ \\
\hline 4.775 & 2.41025 & -5.09 & -35.1 & 1881 \\
\hline 6.35 & 2.4095 & -9.95 & -34.0 & 1557 \\
\hline 7.62 & 2.4085 & -14.9 & -33.7 & 1343 \\
\hline
\end{tabular}


The beam is to be collected on a Faraday cup that has the capability of being biased. The beam collector assembly is shown in Fig. 31, with the Faraday cup separated and shown on the right. Biasing will allow an approximate measurement of the energy and energy spread of the accelerated beam.

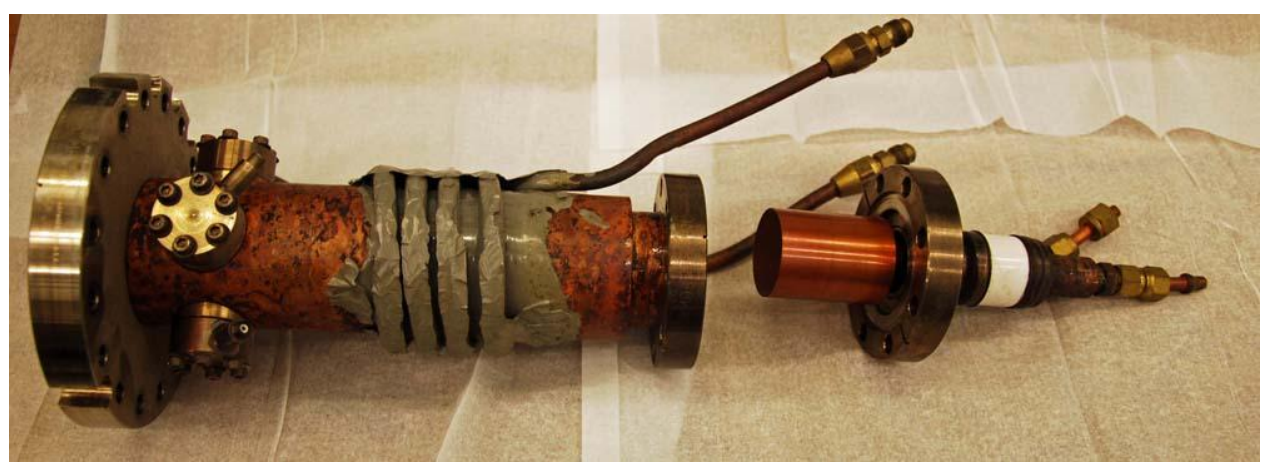

Fig. 31. Beam collector, showing insulated Faraday cup at right.

First experiments with a single cavity should provide beam acceleration, independent of RF phase. Thus even for a continuously injected beam, a sharply-defined group of electrons should be separable from the bulk of the beam using a retarding potential on the collector. Multi-cavity operation, energizing one additional cavity at a time, should provide a beam continuous over the macropulse width only for those particles in the acceptance phase window becoming accelerated through the device. By adjusting the bias on the collector one should be able to determine the percentage of the beam that was accelerated to various energies. Another feature of the Faraday cup setup is that there is a hollow cylindrical obstruction, which for proper settings of the magnetic field in the region leading up to the obstruction, will transmit the full accelerated beam. By adjusting the magnetic field downward the largest energies (largest orbital radii) will be scraped off and not reach the collector. Thus by changing the field, a radial profile of the helical beam can be found.

A second method of beam diagnostic is to replace the Faraday cup with a phosphor screen, of the type shown in Fig. 32. By measuring the light intensity the relative number of accelerated electrons, and their profile, the energy distribution can be determined given the magnetic field strength. 
Omega-P, Inc.

Final Report, DoE SBIR Grant DE-FG02-07 ER 84862

HIGH-INTENSITY PROTON ACCELERATOR

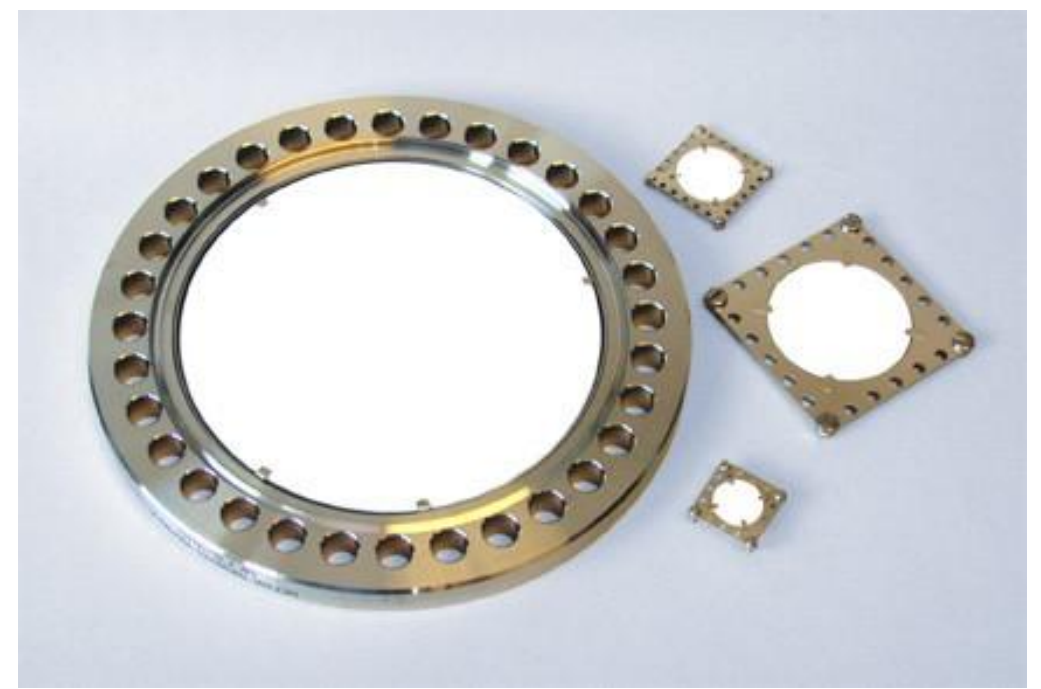

Fig. 32. Phosphor screens of the type to be used for beam profile imaging to determine accelerated beam energy and energy distribution.

\section{ANALYSIS AND TEST OF CAVITY WITH RADIAL VANES}

For a multi-cavity proton cyclotron accelerator, steps on can take to reduce cavity diameters can have a strong influence on system complexity and cost - particularly for the large bore superconducting magnet that will be required. One means of reducing cavity radius is by means of radial vanes, as discussed on pp. 11-12. Fig. 33a and 33b show a four- and eight-vaned cavity analyzed to study this effect. Analysis of this class of $\mathrm{TE}_{111}$-like cavity modes was carried out, including a variety of vane shapes and vane numbers. Attention was paid to field uniformity at the orbit radius, so as to avoid undue phase or energy spreads, and to concomitant reductions in shunt impedance caused by introduction of vanes. Cavities with four and eight vanes,

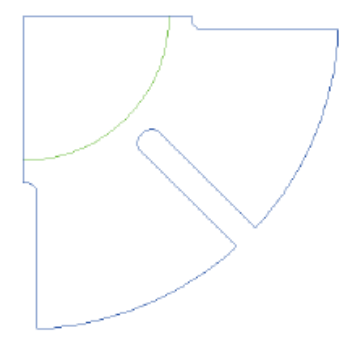

M1

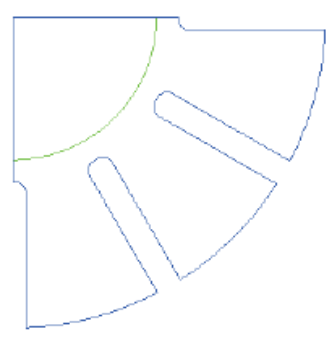

M2

Fig. 33a. Quarter-sections of 4- and 8-vaned cavities, with labels M1 and M2.

labeled M1 and M2 that show the greatest promise for this application because, of all geometries studied, these showed the least reduction in shunt impedance relative to the simple cylinder.. The table below gives, as examples for the 64-MHz cavity in the proton accelerator (the largest cavity - see Table II), some relevant parameters for a simple cylinder as compared with those for M1 and M2. Dimensions for M2 are shown in Fig. 33b. The cavity radii for M1 and M2 are $82 \%$ that for the cylinder, while the shunt impedances are $78 \%$ and $64 \%$ that for the cylinder. 


\section{Omega-P, Inc.}

Final Report, DoE SBIR Grant DE-FG02-07 ER 84862

\section{HIGH-INTENSITY PROTON ACCELERATOR}

For this purpose, the shunt impedance is defined as $(0.9465 \mathrm{~V})^{2} / P$; where the factor 0.9465 is the ratio of the fundamental (dipole) component of the field at the orbit radius $r=65 \mathrm{~cm}$, to the field on axis; where the effective voltage $V$ is the integral of the radial component of RF electric field along the length of the cavity at $r=65 \mathrm{~cm}$; and where $P$ is the power fed to the cavity. The quantities $\mathrm{H}_{2}-\mathrm{H}_{10}$ in Table $\mathrm{V}$ are coefficients of higher

Table. Properties of vaned cavities, compared with a cylinder.

\begin{tabular}{|c|c|c|c|}
\hline & cylinder & M1 & M2 \\
\hline frequency (MHz) & 63.970 & 64.101 & 64.075 \\
\hline radius (cm) & 172 & 141 & 140 \\
\hline shunt impedance $(\mathrm{M} \Omega)$ & 6.94 & 5.44 & 4.41 \\
\hline $\mathrm{H}_{2}$ & 1.00 & 1.00 & 1.00 \\
\hline $\mathrm{H}_{4}$ & $6.67 \times 10^{-2}$ & $1.53 \times 10^{-3}$ & $2.71 \times 10^{-3}$ \\
\hline $\mathrm{H}_{6}$ & $1.19 \times 10^{-3}$ & $1.08 \times 10^{-3}$ & $3.21 \times 10^{-3}$ \\
\hline $\mathrm{H}_{8}$ & $1.04 \times 10^{-3}$ & $1.96 \times 10^{-1}$ & $-3.06 \times 10^{-5}$ \\
\hline $\mathrm{H}_{10}$ & $1.20 \times 10^{-3}$ & $-7.10 \times 10^{-2}$ & $3.19 \times 10^{-3}$ \\
\hline
\end{tabular}
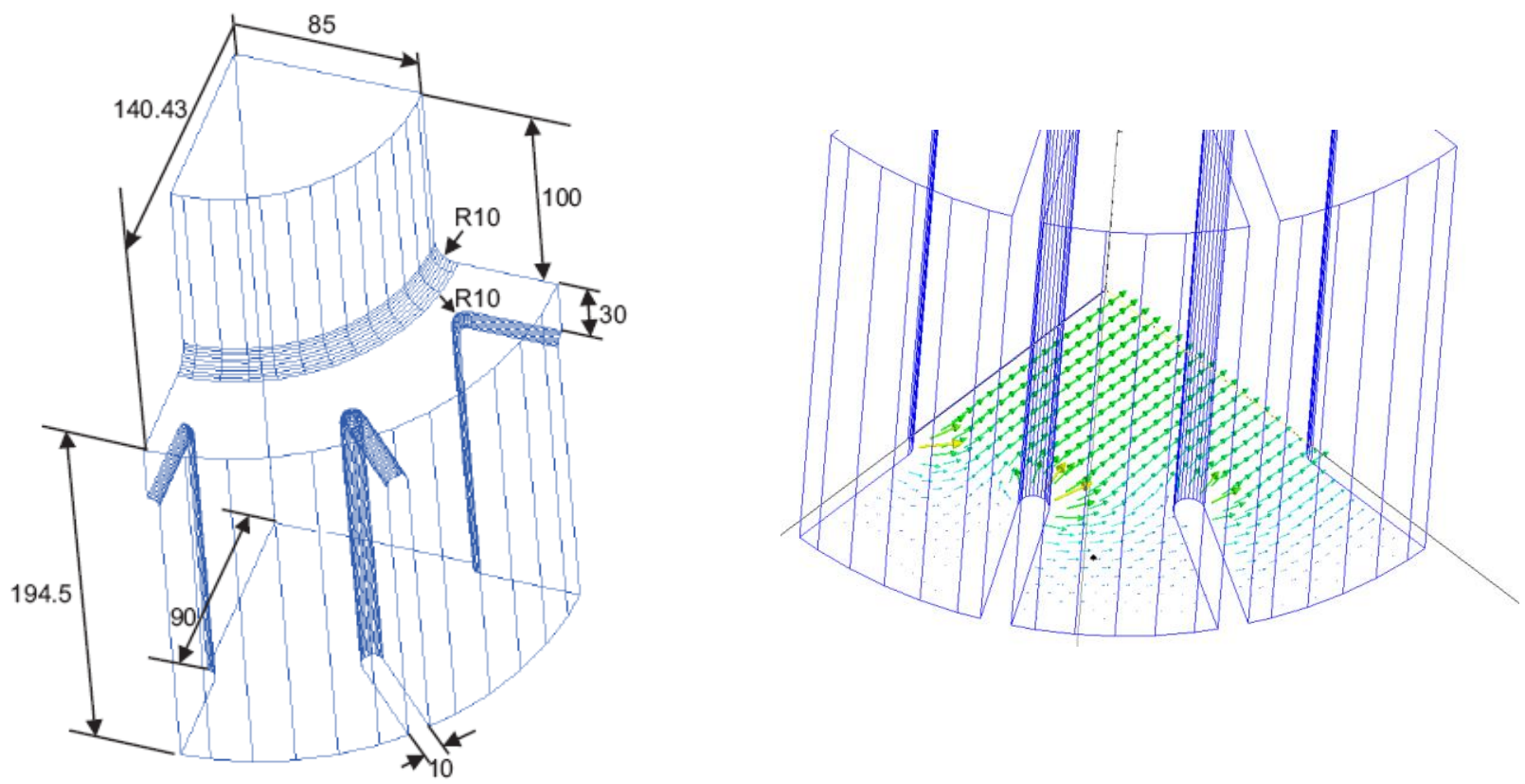

Fig. 33b. One-eighth section of the 8-vane 64-MHz cavity M2. Left figure shows dimensions in $\mathrm{cm}$ for a $64 \mathrm{MHz}$ cavity; right figure shows $E$-field pattern at midplane. Fields are rotating, as a result of using two input coupling loops.

multipole field components seen around the orbit radius imposed by the vanes; it is seen for M2 that departures from the pure dipole field are negligible. Details of loop input coupler are shown in Fig. 19. For excitation of a rotating mode, two such loops are to be used, displaced by $90^{\circ}$ and driven $90^{\circ}$ out of phase. 


\section{Omega-P, Inc.}

Final Report, DoE SBIR Grant DE-FG02-07 ER 84862

HIGH-INTENSITY PROTON ACCELERATOR

The $18 \%$ reduction in cavity radius afforded by use of the vanes should translate into a capital cost savings for the RF system and cryomagnet of at least this order-of-magnitude, while the shunt impedance reductions signify that either $88 \%$ (M1) or $80 \%$ (M2) of the accelerating field will obtain, as compared with the cylinder at the same power level; or that a power increase will be required to achieve the same accelerating fields as in the cylinder. The required power increase depends upon beam loading, i.e. the ratio of power going into the walls compared to that going into the beam. For 67\% efficiency, the required power increase would be about 7\% (M1) or $19 \%$ (M2). Consuming higher power represents a cumulative increase in operating cost, while making up for lower acceleration without increasing power translates into a longer machine with an increase in cost in rough proportion to length. These preliminary considerations show that gain from the use of vaned cavities is a complex issue that should be examined in more detail at the time that a design for a proton machine is under consideration.

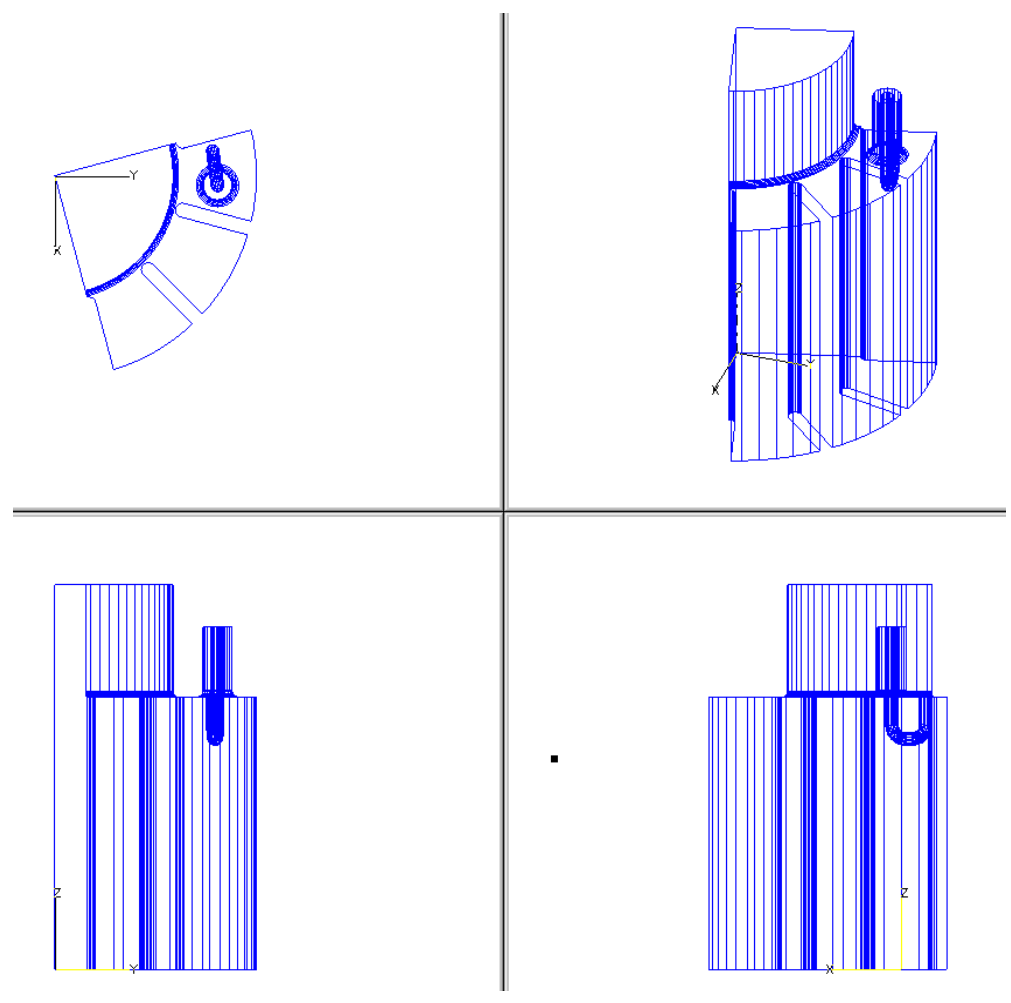

Fig. 34. Disposition of one of the two loops for exciting the cavity. Note that using RF input on cavity side walls does not require additional bore diameter for the magnetic field coils, beyond that needed to accommodate the cavities themselves.

Experiments to test some properties of a cavity of type M2 were carried out on a version scaled to resonate at about $1 \mathrm{GHz}$. Photos of the test cavity are shown in Fig. 35. Cavity dimensions for the bench tests were adjusted for an operating frequency of $1008 \mathrm{MHz}$, so as to keep the dimensions reasonable and the fabrication problems to a minimum. For example, this resulted in a convenient vane thickness of 0.25 ", and all elements were bolted together. Measurements were made using an Agilent model 8714ES vector network analyzer. The cavity 


\section{Omega-P, Inc.}

was driven by magnetic coupling loops, one of which is shown in Fig. 35. The end walls could be rotated so that opposing coupling loops could be displaced at multiples of $45^{\circ}$ intervals from one another. Fig. 36 shows spectral scans of transmission between coupling loops. At the low frequency end of the scan, two single well-separated peaks are seen at approximately 1005 and $1323 \mathrm{MHz}$, regardless of the relative angular displacement of the loops from one another. Above $1400 \mathrm{MHz}$, the spectrum becomes crowded.

The lowest frequency mode singled out and shown in Fig. 37 is the operating mode. It is well separated from any neighboring mode and shows no nearby spurious modes. Its frequency is measured as $1004.935 \mathrm{MHz}$, comparing favorably with the calculated frequency of $1008 \mathrm{MHz}$. Measured $Q$ factors were between 5000 and 6000 depending upon receiver locations; this compares not unfavorably with the calculated value of 9600 , when one takes into account that only bolted construction and not welded joints was used in the aluminum cavity construction.
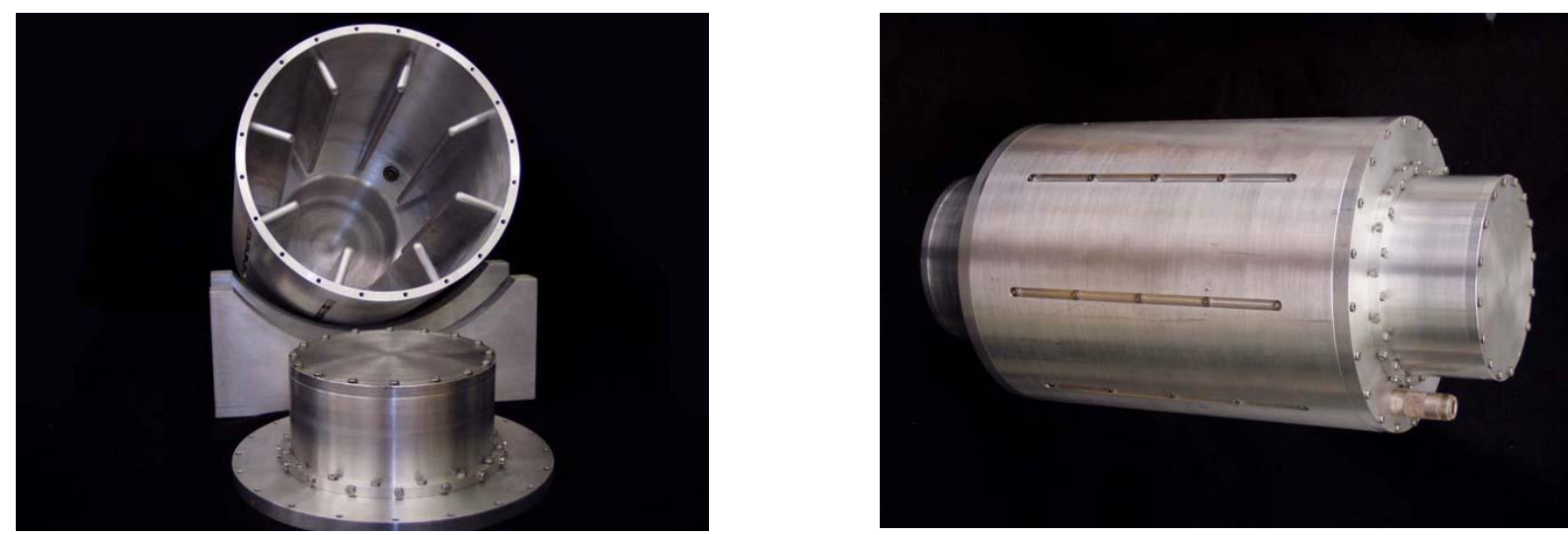

Fig. 35. 1-GHz aluminum cavity of type M2 as tested. Photo on left is with one end wall removed, showing eight radial vanes. Photo of assembled cavity on right shows cutoff drift tubes with cover plates and a connector to one of the two coupling loops.

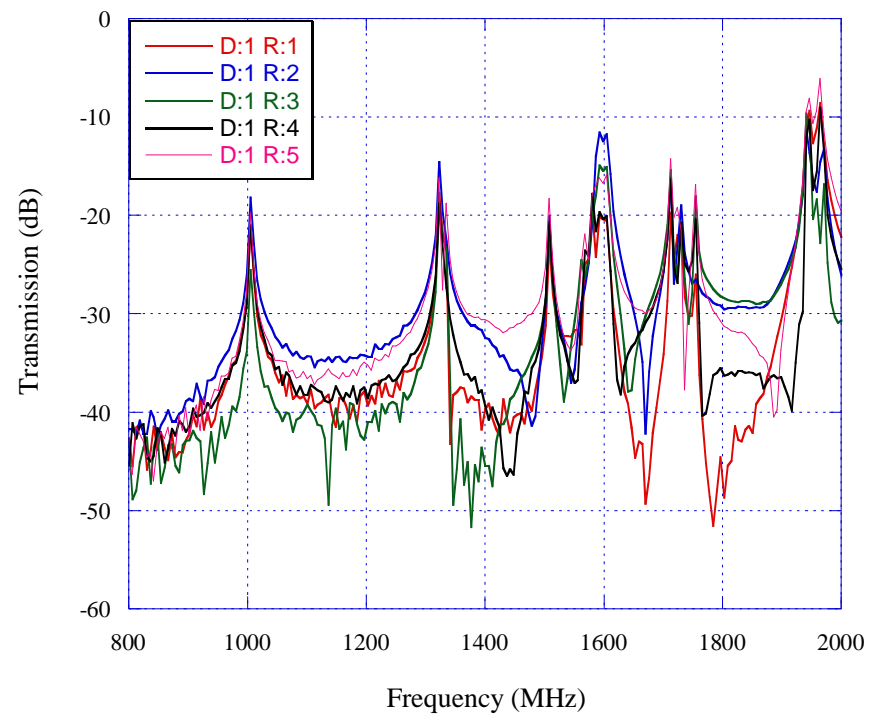

Fig 36. Five transmission traces between coupling loops in the M2 cavity, for the same drive position of the drive loop but differing receiving locations. 


\section{Omega-P, Inc.}

Final Report, DoE SBIR Grant DE-FG02-07 ER 84862

HIGH-INTENSITY PROTON ACCELERATOR

These measurements show that theoretical predictions for the eigenfrequency of the $\mathrm{TE}_{111}$-like operating mode can be confirmed by measurements, that other modes are well separated in frequency, and that unexpected spurious modes are not in evidence. Thus, should it be decided to use internal vanes to reduce the cavity radii in the proton multi-cavity cyclotron accelerator, accurate predictions can be made for the operating frequency and spectrum of competing modes. However, a decision as to whether the benefit of using vaned cavities can outweigh the disadvantage of lowered shunt impedance should be postponed until more is understood as to overall system costs for the proton machine.

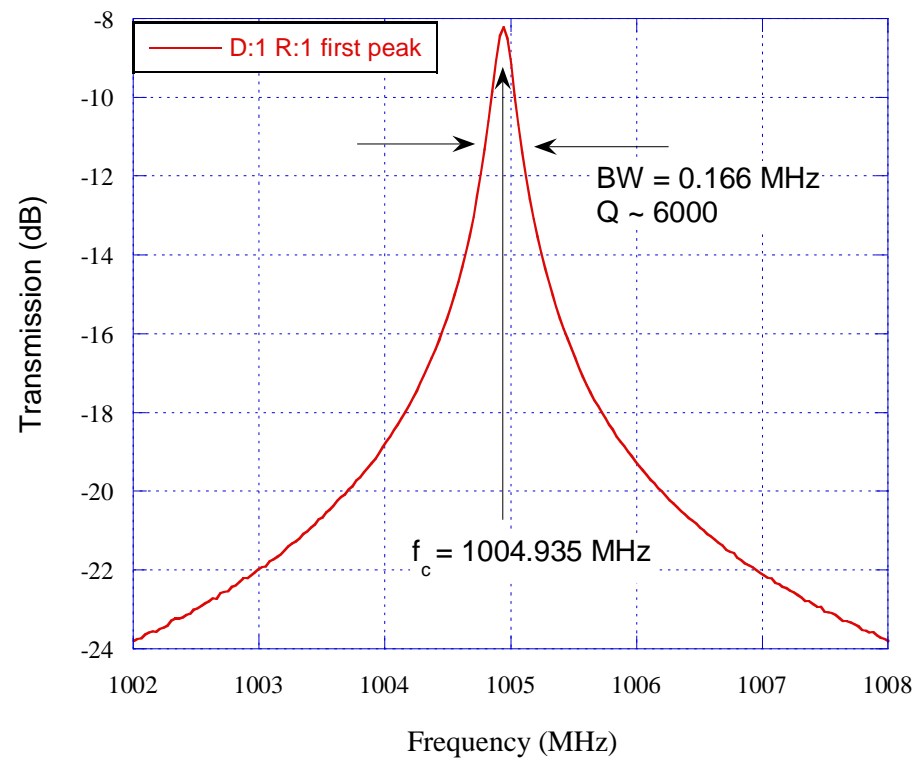

Fig. 37. Narrowband sweep of the lowest mode in the spectrum. This represents the nominal operating mode for the proton accelerator cavities.

\section{TRANSPORT OF A PROTON BEAM FROM A MULTI-CAVITY CYCLOTRON}

Transport of the proton beam after the last cavity in the muti-cavity cyclotron accelerator to a remote target is essential if the beam is to find practical use for almost all applications that are listed in introductory sections of this report. The layout depicted in Fig. 1 implies that the beam target (e.g., an assembly of spent nuclear fuel rods, or fissile fuel within a sub-critical reactor) is positioned adjacent to the accelerator. Considerations of radiation safety, and of needs for routine maintenance of both the accelerator and the target, clearly rule out such proximity. Thus a means for beam transport, probably over a distance of at least several 10's of meters, must be at hand for the proton accelerator to be considered as a serious contender for the applications that have been discussed.

Fig. 3 shows that protons emerge from the last cavity at a large angle to the machine axis, and that the mean direction of emergence is the same for successive bunches. But because the trajectories of each proton bunch are replicated one after another, and because the mean azimuthal bunch position is the same for all the bunches, the proton beam after acceleration may be extracted from the magnetic system and transported to the target using a large-aperture 


\section{Omega-P, Inc.}

Final Report, DoE SBIR Grant DE-FG02-07 ER 84862

\section{HIGH-INTENSITY PROTON ACCELERATOR}

quadruple transport line. The beam azimuthal position may be adjusted and maintained by adjustments in RF phase.

The parameters for focusing elements in a stable transport line can be determined by knowing the beam energy distribution and beam emittance. In the present instance, the beam transverse emittance may be estimated the following way. During the beam acceleration, the longitudinal beam momentum doesn't change significantly, and the energy gain is caused by the particle transverse momentum increase. During the beam acceleration, particles oscillate around the central beam trajectory. The transverse velocity spread is determined, thus, by the beam energy spread. In this case, the transverse emittance of the beam $\varepsilon$ may be estimated by the formula:

$\varepsilon=\sigma_{r} \sigma_{v \perp} / v_{\perp}$,

where $v_{\perp}$ is transverse velocity, $\sigma_{\mathrm{r}}$ is spread in radial coordinate, $\sigma_{v \perp}$ is spread in transverse velocities. Taking into account that

$\sigma_{v \perp} / v_{\perp} \approx \frac{1}{2 \pi}\left(\frac{\sigma_{\gamma}}{\gamma}\right)$

where $\sigma_{\gamma} / \gamma$ is a relative energy spread, and

$\sigma_{r}=r\left(\frac{\sigma_{\gamma}}{\gamma}\right)=\frac{v}{\Omega}\left(\frac{\sigma_{\gamma}}{\gamma}\right)=\frac{\beta U_{0} \gamma}{B c}\left(\frac{\sigma_{\gamma}}{\gamma}\right)$

where $r$ is the orbit radius and $\Omega$ is a cyclotron frequency, one obtains

$\varepsilon \sim \frac{\beta U_{0} \gamma}{2 \pi B c}\left(\frac{\sigma_{\gamma}}{\gamma}\right)^{2}$

where $U_{0}$ is a proton rest mass in $e V$, and $B$ is a guiding magnetic field. For $B=80 \mathrm{kG}$, a relative energy spread of $6 \%$, and $\gamma=2$ (conditions that are predicted after the last cavity), one has $\varepsilon$ $\sim 3 \times 10^{-4} \mathrm{~m}$. Further beam emittance growth caused by the fringing field aberrations should not be significant, because the beam transverse size at the accelerator output is much smaller than the solenoid aperture. In any case, the output beam transverse emittance is large, and in order to transport the beam to the target, large-aperture quadrupole lenses are necessary. Such lenses were developed for example, for FFAG synchrotrons [1], as seen in Fig. 38.

This lens shown in Fig. 38 has a focus distance $F$ of about $6 \mathrm{~m}$ for $1 \mathrm{GeV}$ protons. For a simple FODO lattice with the $L / F=2$ ( $L$ is the distance between the lenses) the maximal $\beta$ function $\beta_{\max }$ is equal to $\sqrt{3} L \approx 20 \mathrm{~m}$, that provides the beam r.m.s. size in the transport line of $\sqrt{\beta_{\max } \varepsilon} \approx 7 \mathrm{~cm}$. These rudimentary estimates show that proton beam transport after acceleration in the multi-cavity proton accelerator can be accomplished using a FODO lattice employing large aperture quads of the sort already built. 


\section{Omega-P, Inc.}

Final Report, DoE SBIR Grant DE-FG02-07 ER 84862

HIGH-INTENSITY PROTON ACCELERATOR

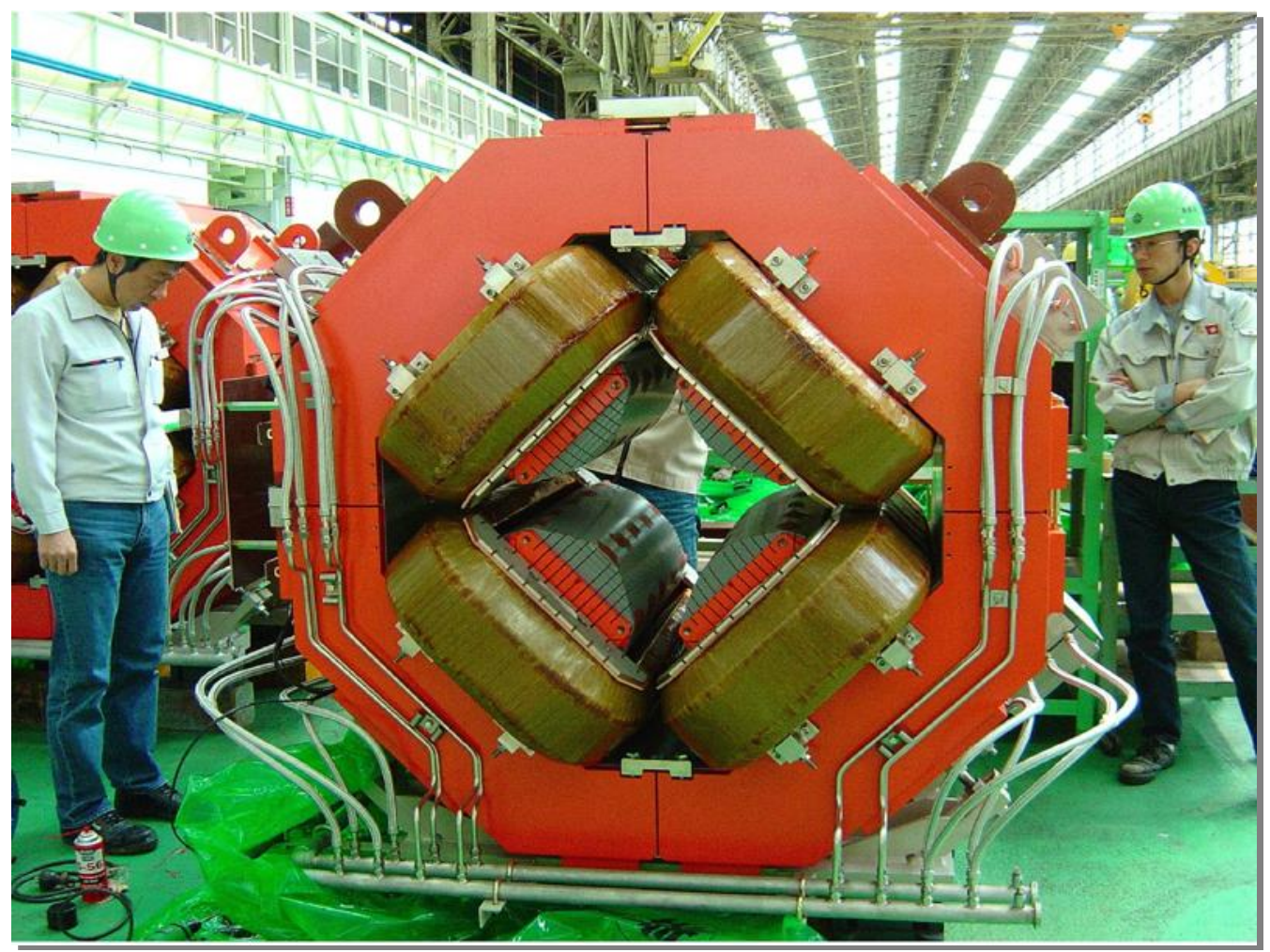

Fig. 38. Large- aperture lens built for FFAG synchrotron.

An actual orbit calculation for an ensemble of protons has been carried out, with results shown in Figs. 39-41. This example is for extraction and focusing from a proton cavity cyclotron of a $210 \mathrm{MeV}$ beam with an initial longitudinal emittance of $52 \mathrm{nsec}-\mathrm{keV}$. Acceptable focusing is seen, with transverse width and height at the focal point of 71 and $61 \mathrm{~mm}$, respectively.

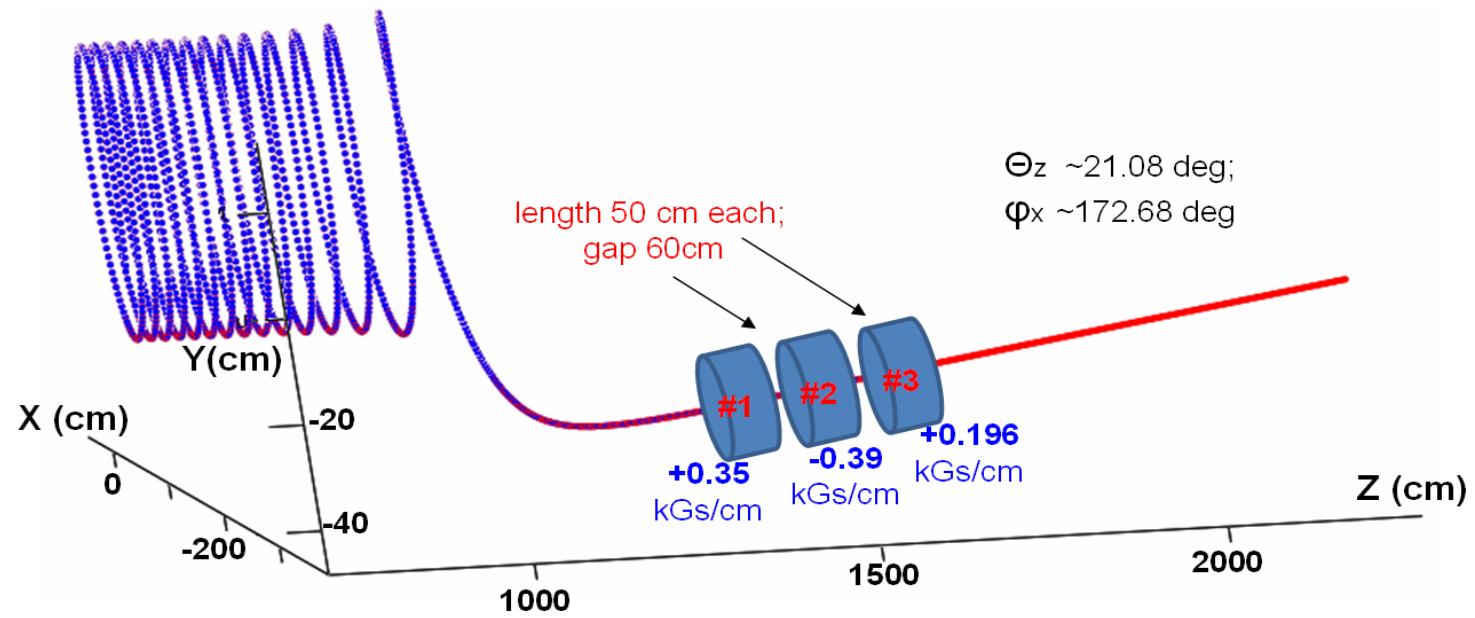

Fig. 39. Orbit for proton beam extracted after the last cavity in the fringing field of the solenoid magnet, and brought to a focus using the arrangement of quads as shown. 
Omega-P, Inc.

Final Report, DoE SBIR Grant DE-FG02-07 ER 84862

HIGH-INTENSITY PROTON ACCELERATOR

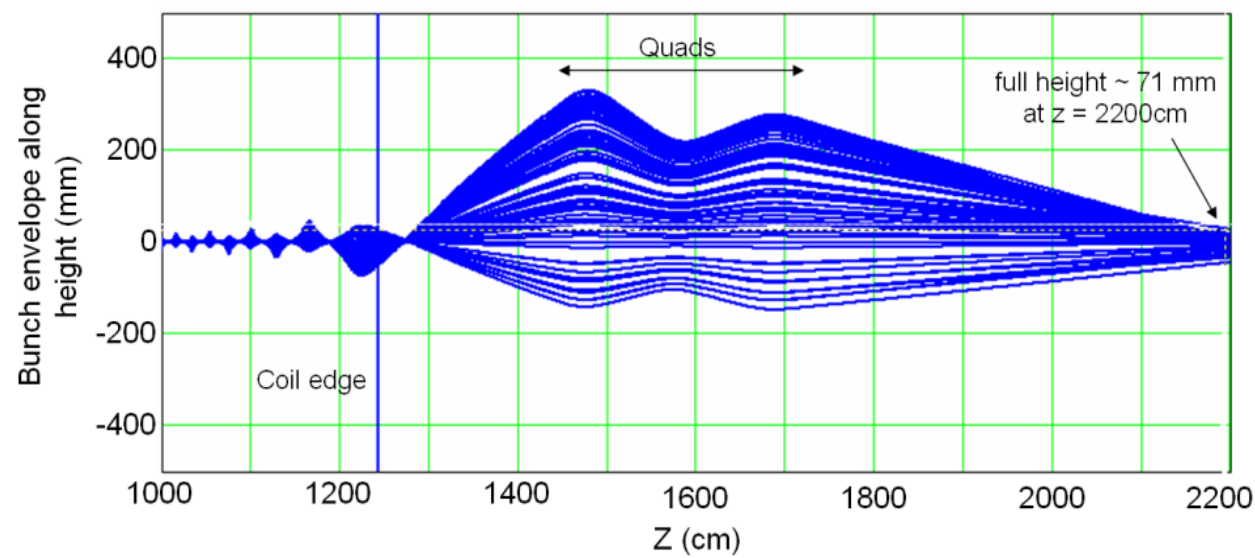

Fig. 40. Development in height of proton beam along the extraction trajectory.

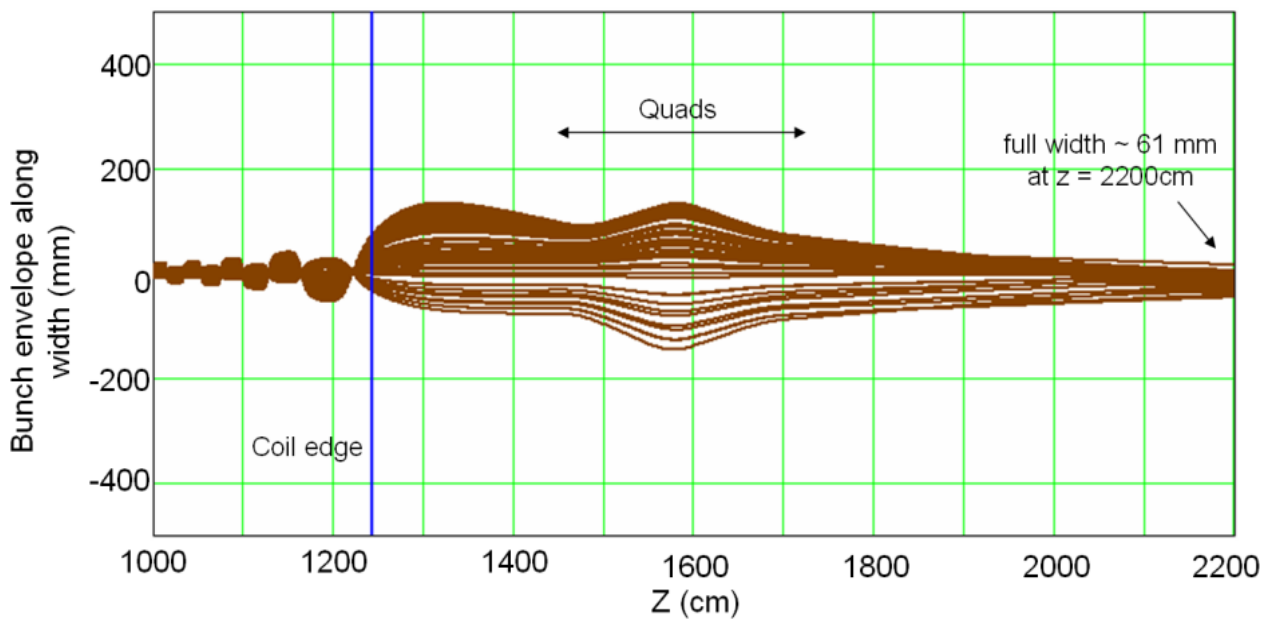

Fig. 41. Development in width of proton beam along the extraction trajectory.

\section{SUMMARY}

Achievements by Omega-P scientists and their collaborators working with support under DoE SBIR grant DE-FG02-07 ER 84862, High Intensity Proton Accelerator, have established a solid understanding of the basic acceleration mechanism in the multi-cavity proton cyclotron accelerator. Early design concerns focused on two important topics: (a) study of vaned cavities to reduce cavity diameters; and $(b)$ considerations for proton beam transport after acceleration. Both topics have received close attention, with conclusions given at the bottom of the list of achievements that follows. More recent emphasis has centered on experimental demonstration, using a multi-cavity electron counterpart, of the underlying acceleration mechanism itself, of the parameters that influence the acceptance phase angle for efficient acceleration without undue emittance growth, and upon achievement of acceptable efficiency for this acceleratorespecially in a high-power room-temperature version as is dictated by the need for a strong axial magnetic field that prevents use of super-conducting RF technology. Achievements within this project include: 


\section{Omega-P, Inc.}

Final Report, DoE SBIR Grant DE-FG02-07 ER 84862

\section{HIGH-INTENSITY PROTON ACCELERATOR}

- Cyclotron-resonance acceleration in each cavity is shown to provide energy gain for the protons. The cavity resonance frequencies $f_{n}$ decrease from $f_{1}$ for the first cavity, with a fixed frequency interval $\Delta f$ between each of the $N$ cavities; thus $f_{n}=f_{1}-(n-1) \Delta f$ and $f_{1}=\ell \Delta f$, with the integer $\ell \geq n$, and $1 \leq n \leq N$. Proton pulses injected into the first cavity at intervals $\Delta T=1 / \Delta f$ or integer multiples thereof can experience continuous energy gain throughout the entire cavity cascade, provided the relative phases for fields in each cavity are properly adjusted.

- Since protons can execute a number of orbital turns in each cavity well in excess of unity, voltage gain can be much larger than the product of peak electric field and cavity length.

- In light of this latter feature, cavity surface fields can be well below breakdown and multipactor limits, even for substantial proton energy gain per cavity.

- Furthermore, since protons are strongly restrained to follow spiral orbits in the strong static magnetic field, additional focusing magnets are not needed, thereby simplifying the design, conserving space, and not diluting the effective acceleration gradient.

- In addition to these distinctive features, it was discovered that this acceleration mechanism can be sustained for exceptionally wide injection phase angles in the first cavity, e.g., two rf cycles in the example shown in this report. This feature is highly significant, in that it allows operation with high duty factor $(\sim 13 \%)$ and low peak proton current $(<1 \mathrm{~A})$, thereby mitigating against issues that can arise from high beam space charge and yet seem to allow high average proton current (>10 mA).

- Simulations were carried out for a preliminary version of a four-cavity electron counterpart, rather than eight cavities as used for the proton case discussed above. This reduction in numbers of cavities is possible since the required power levels for each cavity in the electron accelerator will introduce insignificant cavity wall heat loading.

- Furthermore, for the electron counterpart, the simplest design consistent with demonstration of the essential physics was desired. Parameters were obtained (see Table VI) for a four-cavity electron cyclotron with $8 \mathrm{~mA}$ average current and a $1.0 \mathrm{keV}$ injected beam, i.e., heavy beam loading. The perveance for this beam is $0.25 \times 10^{-6} \mathrm{~A}-\mathrm{V}^{-3 / 2}$.

- For this $8 \mathrm{~mA}$ example, the RF-to-beam efficiency is $71.0 \%$. Cavity frequency separations are $500 \mathrm{MHz}$ and the duty factor is $10 \%$, corresponding to a $0.2 \mathrm{~ns}$ electron pulse injected every 2 ns.

- The injection phase window here is $288^{\circ}$ in the first $(4 \mathrm{GHz})$ cavity. [Simulations show that, the greater the number of cavities for a given acceleration energy, the larger can be the injection phase angle but the greater will be the accelerator length.]

- An electron counterpart four-cavity model of the proton cyclotron accelerator is designed, and is at this writing in cold tests of the cavities. Coils and associated power supplies available at Yale have parameters that limit the design of the cavity system. Balancing competing factors led to the compromise as follows: magnetic field strength $B \approx 1.6 \mathrm{kG}$, and cavity frequencies of $2.4,2.1,1.8$, and $1.5 \mathrm{GHz}$. 


\section{Omega-P, Inc.}

Final Report, DoE SBIR Grant DE-FG02-07 ER 84862

HIGH-INTENSITY PROTON ACCELERATOR

- The magnetic system of coils and pole pieces has been assembled and tested; axial field profile has acceptable uniformity of better than $\pm 2 \%$. Each coil is powered from its own dedicated power supply.

- Cavity system is of necessity of demountable design, so as to permit tuning of cavities individually by machining radii and lengths as needed, and to allow convenient access ports for four coaxial feed and sampling antennas in each cavity, without need for multiple iterations in fabrication and associated excessive costs that would attend use of a one-piece brazed assembly.

- As a consequence, the unloaded cavity $Q$ factors were lower than for a design without demountable joints. This in turn led to a decision not to operate the first prototype of the accelerator in a range where beam loading would further lower the $Q$ factors, but to choose a design with 10-50 $\mu \mathrm{A}$ current. Electron gun has been built.

- This choice of low current prevents measurements of the ultimate efficiency of the accelerator, but would permit determination of the less-understood phase acceptance of the multi-cavity acceleration mechanism - a phenomenon that has a direct bearing on the efficiency as well as the beam quality.

- Cavity coupling and tuning have been measured, and a prescription is determined for reducing the present $3 \%$ power reflected and $\sim 8 \mathrm{MHz}$ tuning error for the $2.4 \mathrm{GHz}$ cavity.

- A four-frequency RF system has been built and tested, with amplifiers that supply at least $500 \mathrm{~W}$ each of phase synchronized pulsed power at 1.5, 1.8, 2.1, and $2.4 \mathrm{GHz}$. Measurements of phase control and stability have shown acceptable performance.

- First experiments with the electron counterpart are to be with only the first $(2.4 \mathrm{GHz})$ cavity and the beam collector, so as to check beam acceleration and confirm utility of RF and beam diagnostics. Tests with all four cavities are to follow.

- Cavities for an eventual proton machine with a wide range of vane configurations were analyzed and one (M2) was selected, having good uniformity in accelerating field at the orbit radius, and having the least diminution in shunt impedance, as compared with that for a corresponding cylindrical cavity.

- Field distributions and loop coupling details were produced for cavity M2.

- An eight-vaned aluminum test version of cavity M2 was built, with an operating mode resonance frequency of about $1 \mathrm{GHz}$, as compared with $64 \mathrm{MHz}$ for the lowest frequency cavity in the proton machine.

- The measured resonance frequency and quality factor $Q$ were in good agreement with the calculated value.

- No clear preference emerged as to choice of vaned cavities for a future proton machine, given the conflict between smaller cavity and magnet coil bore-with attendant lower capital cost-and lower cavity shunt impedance-with attendant lower acceleration gradient and/or a longer machine. 


\section{Omega-P, Inc.}

Final Report, DoE SBIR Grant DE-FG02-07 ER 84862

\section{HIGH-INTENSITY PROTON ACCELERATOR}

- No present justification exists for using vaned cavities in the electron counterpart machine.

- Beam emittance after acceleration was calculated.

- Emittance and beam energy spread were used to estimate the required aperture for quadrupole magnets needed in a beam transport lattice to convey the beam from the accelerator to a remote target.

- The required large aperture quadrupoles appear to be within the existing state-of-the-art for non-superconducting magnets.

- A transport and focusing system was devised for the proton beam exiting the final cavity in a three-cavity example. Beam trajectory in the diverging magnetic field of the main solenoid was computed, and acceptable focusing using three quads was demonstrated in the computed trajectories. 


\section{REFERENCES}

1. "The Future Path for Advanced Spent Fuel Treatment and Transmutation Research: Report to Congress on the Advanced Fuel Cycle Initiative (AFCI)," Office of Nuclear Energy, Science and Technology, US Department of Energy, January 2003.

2. J. L. Hirshfield, C. Wang, and R. Symons, "Multi-stage, high-gradient, cyclotron resonance proton accelerator concept," in Advanced Accelerator Concepts: $9^{\text {th }}$ Workshop, edited by P. L. Colestock and S. Kelley, AIP Conf. Proc. 569 (AIP, New York, 2001) pp. 833-843; US Patent \#6,914,396 B1 "Multi-stage cavity cyclotron resonance accelerator,” R.S. Symons, J.L. Hirshfield, C.B. Wang, inventors, July 5, 2005.

3. C. Wang and J. L. Hirshfield, "Simulation study of multi-stage proton cyclotron resonance accelerator - PROCRA," in Proceedings of the 2001 Particle Accelerator Conference, June 1822, Chicago, Illinois, edited by P. Lucas and S. Webber (IEEE, NJ, 2001), pp. 3389-3391.

4. J. L. Hirshfield, C. Wang, and R. Symons, "Multi-stage cyclotron resonance proton accelerator," in Proc. 2001 ANS Winter Meeting, Nov. 11-15, 2001, Reno, Nevada.

5. J. L. Hirshfield, C. Wang, and V. P. Yakovlev, "Multicavity proton cyclotron accelerator," Phys. Rev. STAB 5, 081301 (2002).

6. J. L. Hirshfield, C. Wang, V. P. Yakovlev, and R. S. Symons, "Multi-cavity proton cyclotron accelerator," Proc. $11^{\text {th }}$ Int. Conf. on Emerging Nuclear Energy System, 29 Sept. - 4 Oct., 2002. pp. 92-100 (2003).

7. Proc. of the $15^{\text {th }}$ International Conference on Magnet Technology (MT-15), Beijing, China (October 20-25, 1997). Large superconducting magnets include: 6-T, 1.19-m ID, 4.9-m long MHD coil for Coal-Fired Flow Facility (168 MJ); 9.2-T, 7.8-m ID Large Helical Device coil for NIFS, Japan (1,700 MJ); and 13-T, 1.6-m ID, 2.8-m long central solenoid model coil for ITER (650 MJ). Construction is underway for a 13-T, 3.8-m ID, 5.4-m long central solenoid coil for ITER (13,300 MJ). Other examples are a 3.8-T, 9.4-m ID, 26-m long barrel magnet for the ATLAS detector, LHC; 4.6-T, 5.4-m ID, 13-m long coil for compact muon collider; and a 13-T, 11(16)-m ID 50-m toroidal coil for ITER (103,000 MJ). (Stored energy is given in parentheses).

8. V. P. Yakovlev, O.A. Nezhevenko, O.V. Danilov, D.G. Myakishev, and M.A. Tiunov, "State of the Art Simulations of Magnicon," in Advanced Accelerator Concepts: $10^{\text {th }}$ Workshop, edited by C. E. Clayton and P. Muggli, AIP Conf. Proc. 647 (AIP, New York, 2002) pp. 421-432.

9. Shinji Machida, "Muon Acceleration and FFAG," NuFact05 Summer Institute, KEK, June 12-20, 2005.

10. R. Raja, at http://ppd.fnal.gov/experiments/e907/raja/energy_amplifier/raja_ea.pdf (2007). 